\title{
CHEMICAL EVOLUTION OF STAR-FORMING REGIONS
}

\author{
Ewine F. van Dishoeck \\ Leiden Observatory, PO Box 9513, 2300 RA Leiden, The Netherlands; \\ e-mail: ewine@strw.leidenuniv.nl \\ Geoffrey A. Blake \\ Division of Geological and Planetary Sciences, California Institute of Technology, \\ Mail Stop 150-21, Pasadena, California 91125; e-mail: gab@gps.caltech.edu
}

KEY WORDS: interstellar medium, astrochemistry, interstellar molecules, star formation, molecular processes

\begin{abstract}
Recent advances in the understanding of the chemical processes that occur during all stages of the formation of stars, from the collapse of molecular clouds to the assemblage of icy planetesimals in protoplanetary accretion disks, are reviewed. Observational studies of the circumstellar material within 100-10,000 AU of the young star with (sub)millimeter single-dish telescopes, millimeter interferometers, and ground-based as well as space-borne infrared observatories have only become possible within the past few years. Results are compared with detailed chemical models that emphasize the coupling of gas-phase and grain-surface chemistry. Molecules that are particularly sensitive to different routes of formation and that may be useful in distinguishing between a variety of environments and histories are outlined. In the cold, low-density prestellar cores, radicals and long unsaturated carbon chains are enhanced. During the cold collapse phase, most species freeze out onto the grains in the high-density inner region. Once young stars ignite, their surroundings are heated through radiation and/or shocks, whereupon new chemical characteristics appear. Evaporation of ices drives a "hot core" chemistry rich in organic molecules, whereas shocks propagating through the dense envelope release both refractory and volatile grain material, resulting in prominent $\mathrm{SiO}, \mathrm{OH}$, and $\mathrm{H}_{2} \mathrm{O}$ emission. The role of future instrumentation in further developing these chemical and temporal diagnostics is discussed.
\end{abstract}




\section{INTRODUCTION}

The combination of rapidly improving observational tools and increasingly sophisticated theory has provided, for the first time, a broad outline of the physical processes associated with the assembly of Sun-like stars and their attendant planetary systems (cf Shu et al 1993, Beckwith \& Sargent 1996, Li $\&$ Shu 1996). Figure 1 presents an overview of the scenario developed for the formation of a single, isolated low-mass star from the collapse of a molecular cloud core. Despite these impressive gains, many crucial details remain poorly understood. In addition, the scope of the problem of stellar and planetary formation has broadened drastically with the discovery of massive extrasolar planets (cf Mayor \& Queloz 1995, Marcy \& Butler 1996). Furthermore, stars are by their nature gregarious. Whether they are part of a multiple system or members of an association, the majority of stars are born in environments that are considerably more complex than that outlined in Figure 1. The characterization of star-forming regions therefore presents considerable challenges both observationally and theoretically.

To this table chemistry does not arrive empty handed. Indeed, observations of molecules play a pivotal role in understanding the physical and chemical evolution of star-forming molecular cloud cores and primitive solar systems. This occurs because the tremendous range in physical conditions and size scales that are present in young stellar objects (YSOs), where densities from $10^{4}$ to $>10^{13}$ molecules $\mathrm{cm}^{-3}$ and temperatures of 10-10,000 K exist over distances from a few stellar radii to many thousands of astronomical units (AU), is perhaps best probed by molecular spectroscopy. Molecules also provide direct access to the velocity fields present in cloud cores with hundreds to thousands of magnitudes of extinction, and their abundances give constraints on the internal and external radiation fields. A significant fraction of the molecules is condensed in icy mantles on dust grains, which contain important information on the temperature and irradiation history of the region. Finally, because chemistry controls critical physical parameters in star formation such as the fractional ionization and cooling of the gas, a detailed understanding of the chemical composition of the gas and dust surrounding young stars is important and interesting in its own right.

Figure 1 A schematic view of low-mass star formation. (a) Dark cloud cores, roughly $1 \mathrm{pc}$ in size, gradually contract until $(b)$ magnetic support is overcome and inside-out collapse begins at $t=0$. (c) For $\sim 10^{4}-10^{5}$ years, a phase of both high accretion and supersonic outflow occurs in deeply embedded protostars (young stellar objects or YSOs). (d) Gradual clearing by the outflow leaves only the young $\mathrm{T}$ Tauri star and a residual protoplanetary accretion disk, which, on time scales of $10^{6}-10^{7}$ years, leads to the formation of a mature planetary system $(e)$. Characteristic molecules at each of these stages are indicated (Figure by MR Hogerheijde, after Shu et al 1987). 


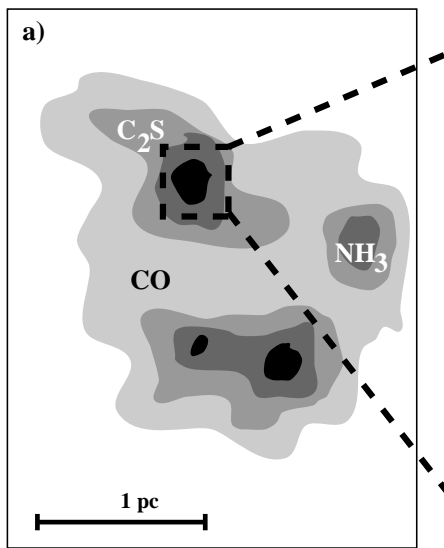

b)

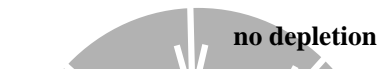

c)

d)
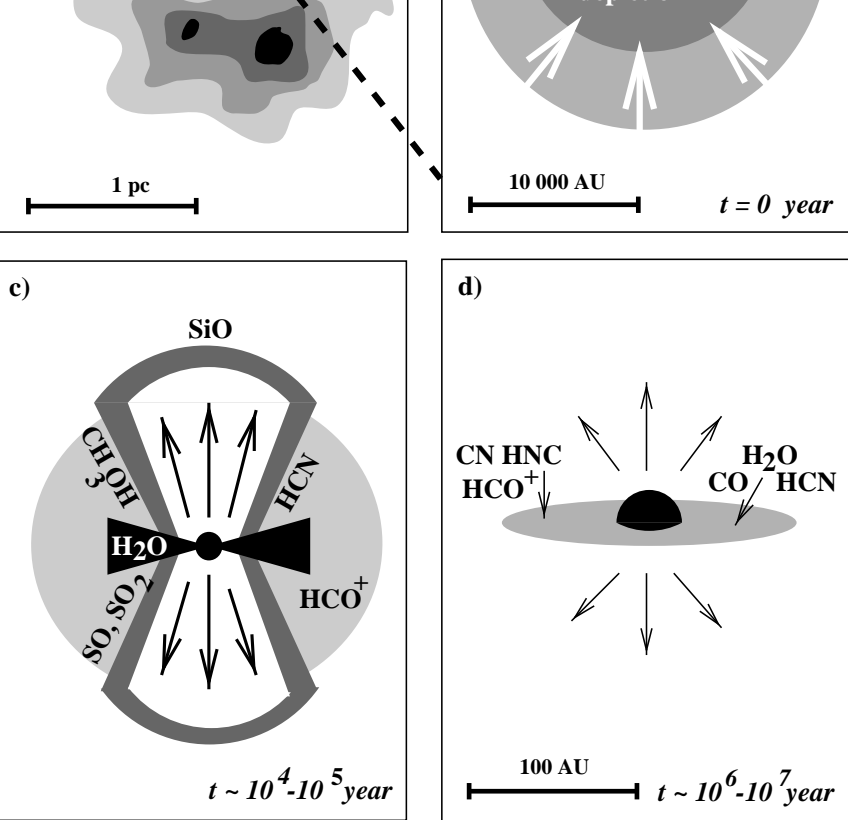

ices $\left(\mathrm{H}_{2} \mathrm{O}, \mathrm{CO}, \mathrm{CO}_{2}\right)$

depletion

)
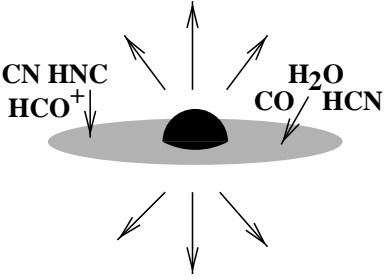

$100 \mathrm{AU}$ $t \sim 10^{6}-10^{7}$ year

e)

$\mathrm{H}_{2} \mathrm{O}, \mathrm{CH}_{3} \mathrm{OH}$,

$\mathrm{H}_{2} \mathrm{CO}, \mathrm{HCN}, \ldots$

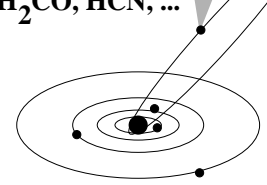

$50 \mathrm{AU}$

$t>10^{7}$ year 
Over the last decade, a scenario has emerged for the main chemical processes that occur during star formation (see Irvine et al 1987, van Dishoeck et al 1993, Hartquist et al 1993, 1998, van Dishoeck \& Blake 1995, Blake 1997, Tielens \& Whittet 1997 for reviews). In cold molecular cloud cores prior to star formation, the chemistry is dominated by low-temperature gas-phase ion-molecule and neutral-neutral reactions leading to the formation of small radicals and unsaturated molecules. During the cold collapse phase, the density becomes so high that most molecules accrete onto the grains and form an icy mantle. Here the chemistry can be actively modified by surface reactions and through processing by ultraviolet photons, $X$ rays, and cosmic rays. After the new star has formed, its radiation heats up the surrounding gas and dust and the molecules begin to evaporate back into the gas phase, with the most volatile species returned first. In addition, the outflows from the young star(s) penetrate the surrounding envelope, creating high-temperature shocks and lower-temperature turbulent regions in which the icy mantles and more refractory material containing silicon can be returned to the gas. These freshly evaporated molecules can then drive a rich and complex chemistry in the gas, called the hot core phase, for a period of $\sim 10^{5}$ years. Finally, the envelope is dispersed by winds and, in the case of massive stars, ultraviolet photons, leading to the appearance of photon-dominated regions (Hollenbach \& Tielens 1997). Thus, significant evolution is expected to occur in the chemical abundances, and this review discusses each of these stages in some detail.

Another important question is in what chemical compounds the major elements $(\mathrm{O}, \mathrm{C}, \mathrm{N}$, etc) are incorporated into forming planetary systems, and the first observations that directly probe the chemistry of circumstellar disks are mentioned here. The wealth of new data on the composition of cometary ices is compared with that found in star-forming regions, and the strong resemblance greatly strengthens the link between the outer-Solar System and interstellar material. The chemistry in the inner solar nebula and its relationship to planetesimals such as meteorites has been reviewed most recently by Lunine (1997) and are not discussed here.

Because of space limitations, it is not possible to provide herein a comprehensive overview of interstellar chemistry and star formation. Only a roadmap through the vast literature on this subject is given and the most characteristic aspects of the chemical evolution during star formation highlighted. Indeed, one of the main long-term goals of astrochemistry is to use certain molecules as "signposts" of the evolutionary state of an object, where enhancements or decreases of their abundances compared with those in pre-star-forming clouds act as temporal indicators. These chemical diagnostics can then be compared with other, more traditional indicators such as the development of an $\mathrm{H} \mathrm{II} \mathrm{re-}$ gion or the size and time scale of the outflow. Such a broad approach runs the 
risk of oversimplification, and many of the subtle difficulties are not mentioned explicitly. The reader is encouraged to consult the original literature and more extensive reviews as much as possible. Good starting points are Herbst (1995), along with the books edited by Millar \& Williams (1993), van Dishoeck (1997), and Hartquist \& Williams (1998).

\section{OBSERVATIONAL TECHNIQUES}

The chemical composition of the highly extinguished circumstellar environment can be probed by infrared and (sub)millimeter techniques, which give very complementary information (Evans et al 1991, Carr et al 1995, Sargent \& Welch 1993). Traditionally, most of the chemical information has been derived from (sub)millimeter single-dish observations. Early millimeter telescopes had typical beam sizes of $1^{\prime}$, corresponding to linear scales of nearly 10,000 $\mathrm{AU}$ $(0.04 \mathrm{pc})$ in the nearest low-mass star-forming regions in Taurus and Ophiuchus. Thus, most of the older literature is primarily concerned with the chemical composition of the lower-density surrounding cloud. With the advent of larger submillimeter telescopes and sensitive high-frequency receivers, more direct probing of the dense envelopes surrounding the YSOs has become possible on scales of $\sim 10-20^{\prime \prime}$ (1500-3000 AU). However, even on this scale, many different physical processes occur (infall, outflow, formation of accretion disks; see Figure 1) that are "blurred" together in the single-dish data. Interferometer observations on scales of $1^{\prime \prime}$ (150 AU) or less are essential to disentangle the different chemical components, but no extensive chemical surveys have yet been done with these instruments, except for the brightest high-mass region, Orion-KL (Murata et al 1992, Minh et al 1993, Blake et al 1996, Wright et al 1996). Most of the chemical scenario presented in this review therefore is based on single-dish data on scales of a few thousand AU (nearby low-mass regions) to $0.1 \mathrm{pc}$ (more distant high-mass regions), with selected interferometric imaging of prominent species.

Infrared observations probe the absorption of material along the line of sight to the embedded young star. This technique has the advantage that only a pencil-beam line of sight is sampled. Also, the population distribution over the rotational energy levels can be directly constrained from a single rovibrational infrared spectrum, whereas often different receivers or telescopes are needed to determine the excitation from (sub)millimeter data. The excitation temperature provides information on the physical parameters of the gas where the molecule is located. In addition, important molecules without a permanent dipole moment such, as $\mathrm{H}_{3}^{+}, \mathrm{CO}_{2}, \mathrm{CH}_{4}$, and $\mathrm{C}_{2} \mathrm{H}_{2}$, possess strong infrared rovibrational transitions but negligible millimeter rotational emission. Finally, both gas-phase molecules and solid-state species can be detected at infrared 
wavelengths, where the latter can be distinguished because their spectra consist of a single broad spectral feature that lacks the characteristic rovibrational structure of the gas-phase spectrum (see Section 7.4).

The infrared solid-state data provide more than just abundances. The shape and position of the ice bands are sensitive to the intermolecular interactions therein (e.g. Sandford et al 1988, Ehrenfreund et al 1997a). For example, CO surrounded by polar, $\mathrm{H}$-rich molecules $\left(\mathrm{H}_{2} \mathrm{O}, \mathrm{CH}_{3} \mathrm{OH}\right.$, etc) has a broader line shape that is shifted to the red because of the formation of hydrogen bonds from that of $\mathrm{CO}$ embedded in a nonpolar, H-poor matrix $\left(\mathrm{O}_{2}, \mathrm{~N}_{2}\right.$, etc). Such shifts have been observed and provide information on the chemical differentiation along the line of sight (e.g. Tielens et al 1991, Chiar et al 1995). In addition, the temperature history of the ices is reflected in the band profiles (e.g. Smith et al 1989). Enormous progress has been made in infrared observations in the last five years owing to the development of sensitive ground-based infrared spectrographs, as well as the launch of the Infrared Space Observatory (ISO). The Short Wavelength Spectrometer (SWS) aboard ISO with a resolving power $\lambda / \Delta \lambda \sim 2000$ is particularly well suited for the study of solid-state features (Whittet et al 1996; see Figure 2).

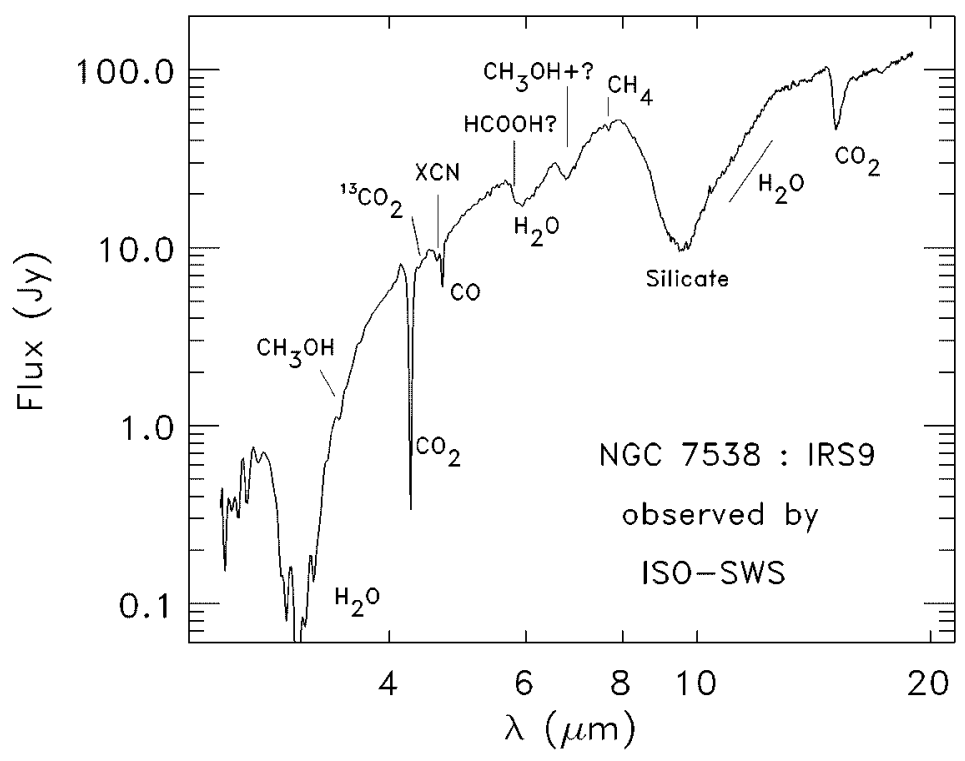

Figure 2 Infrared Space Observatory (ISO)-Short Wavelength Spectrometer (SWS) spectrum of the deeply embedded massive YSO NGC 7538 IRS9, covering the entire 2- to $20-\mu \mathrm{m}$ region. A variety of ice mantle and refractory grain core features are evident. Labels of the most intense features are presented for clarity (Whittet et al 1996). 
On the other hand, submillimeter data have the advantage that they are not restricted to absorption toward the infrared source but can map the surroundings equally well. Also, the spectral resolving power is much higher $\left(R \geq 10^{6}\right)$ so that the line profiles are kinematically well resolved and provide information on the location of the molecule (e.g. outflow versus envelope). Finally, molecules with much lower abundances can be detected with millimeter emission techniques, down to $10^{-11}$ with respect to hydrogen. For comparison, the ISO infrared data probe abundances down to $\sim 10^{-7}-10^{-8}$.

For both submillimeter and infrared observations, the derived chemical abundances may have considerable uncertainties, owing to uncertainties in the excitation, optical depth of the lines, and unresolved source structure (e.g. Irvine et al 1985, 1987). In general, a good physical model of the source is a prerequisite for deriving accurate abundances: Strong lines do not necessarily reflect higher abundances but can also be due to higher densities or temperatures. Another uncertainty is the fact that no direct observations of $\mathrm{H}_{2}$ are generally available for the same line of sight. Several methods have been developed to infer $N\left(\mathrm{H}_{2}\right)$ indirectly (see van Dishoeck \& Black 1987, van Dishoeck 1998a for reviews). Indeed, the only direct determination of $\mathrm{CO} / \mathrm{H}_{2}=2.7 \times 10^{-4}$ in one (warm) dense cloud by Lacy et al (1994) is a factor of three higher than the value of $\mathrm{s} 8 \times 10^{-5}$ commonly used in dark clouds (Frerking et al 1982). In the coldest densest regions, even CO may be significantly condensed into the solid phase, and for such objects, other molecules like $\mathrm{HCO}^{+}$may provide robust tracers of $\mathrm{H}_{2}$ once their abundances are better understood (Pratap et al 1997, Hogerheijde et al 1997a). Given these uncertainties, comparison between observations and models is usually performed at the level of factors of a few. Since the dynamic range in measured abundances is orders of magnitude, this does not pose limitations for the species considered here.

\section{INTERSTELLAR CHEMISTRY}

\subsection{General Characteristics}

Interstellar clouds are the sites of a very rich chemistry, as evidenced by the detection of nearly 120 different molecules (see Ohishi 1997 for a recent summary; links to an up-to-date list of detected molecules and molecular databases can be found through the IAU working group on astrochemistry $<$ http://www.strw.leidenuniv.nl/ $\sim$ iau $34>$ ). The observed species range from simple diatomic molecules in diffuse clouds to long, unsaturated carbon chains in dark pre-star-forming clouds and saturated organic species near massive YSOs. Many models have been developed since the 1940s to describe the chemistry in interstellar clouds, ranging from pure ion-molecule gas-phase networks driven by cosmic-ray ionization (e.g. Bates \& Spitzer 1951, Herbst \& Klemperer 1973) to pure grain-surface chemistry models (e.g. Hollenbach \& 
Salpeter 1971, Allen \& Robinson 1977, Tielens \& Hagen 1982). Modern networks contain up to 4000 different reactions among several hundred species (Lee et al 1996a, Millar et al 1997a). The basic gas-phase molecular processes have been reviewed by Dalgarno (1987) and van Dishoeck (1988), whereas the chemical networks have been described extensively in the literature, e.g. by Watson (1978), Prasad et al (1987), Turner \& Ziurys (1988), Turner (1989), Millar (1990), Winnewisser \& Herbst (1993), Herbst (1995), and van Dishoeck (1998a,b), and in the books by Duley \& Williams (1984) and Bakes (1997).

Ion-molecule gas-phase schemes have been remarkably successful in explaining many observational aspects of interstellar chemistry (Herbst 1997), including the abundances of simple hydrocarbons in diffuse and translucent clouds, the presence of $\mathrm{H}_{3}^{+}$(Geballe \& Oka 1996), and the related high abundances of protonated ions such as $\mathrm{HCO}^{+}$and $\mathrm{N}_{2} \mathrm{H}^{+}$, the high abundances of unsaturated (e.g. $\mathrm{HC}_{7} \mathrm{~N}$ ) and metastable (e.g. $\mathrm{HNC}$ ) species in dark clouds, and the high isotopic fractionation effects found in pairs such as $\mathrm{DCO}^{+} / \mathrm{HCO}^{+}$and DCN/HCN (Wootten 1987, Millar et al 1989). On the other hand, it has become clear that gas-phase chemistry by itself cannot explain all of the data and that gas-grain interactions and grain-surface chemistry are essential, as is evidenced by the detection of ices (Figure 2) and the high abundances of $\mathrm{NH}_{3}$ and $\mathrm{H}_{2} \mathrm{~S}$ in some dark clouds (e.g. Turner 1996) or of $\mathrm{CH}_{4}$ (Boogert et al 1996, 1998) and saturated organic molecules such as $\left(\mathrm{CH}_{3}\right)_{2} \mathrm{CO}, \mathrm{CH}_{3} \mathrm{COOH}$, and $\mathrm{C}_{2} \mathrm{H}_{5} \mathrm{CN}$ in warm star-forming regions (Snyder 1997). The composition of ice mantles has recently been reviewed by Tielens \& Whittet (1997).

\subsection{Gas-Phase Chemistry}

A few basic points about interstellar chemistry are useful to reiterate for the purposes of this review. First, the abundances of the elements play a key role, especially the absolute and relative abundances of carbon and oxygen in atomic form and in all other forms in the gas phase. Because hydrogen is so much more abundant than any other element, reactions with $\mathrm{H}$ and $\mathrm{H}_{2}$ dominate the networks if they are exothermic. This is only the case for small ions; most reactions of neutrals and large ions with $\mathrm{H}$ or $\mathrm{H}_{2}$ have substantial energy barriers, so they do not proceed at the low temperatures in pre-starforming cores and collapsing envelopes. In hot core regions and shocked gas, however, some become significant, especially the $\mathrm{O}+\mathrm{H}_{2} \rightarrow \mathrm{OH}$ and $\mathrm{OH}+$ $\mathrm{H}_{2} \rightarrow \mathrm{H}_{2} \mathrm{O}$ reactions that drive most of the oxygen into water above $\sim 230 \mathrm{~K}$ (Charnley 1997).

The production of complex hydrocarbons in cold clouds requires the presence of atomic carbon as either $\mathrm{C}^{+}$or $\mathrm{C}$, and it occurs via three types of gas-phase pathways (Herbst \& Leung 1989, Herbst 1995): (a) carbon insertion reactions (e.g. $\mathrm{C}^{+}+\mathrm{CH}_{4} \rightarrow \mathrm{C}_{2}+\mathrm{H}_{2} \mathrm{H}_{2}^{+}$or $\mathrm{C}+\mathrm{C}_{2} \mathrm{H}_{2} \rightarrow \mathrm{C}_{3} \mathrm{H}+\mathrm{H}$ ); (b) condensation 
reactions (e.g. $\mathrm{CH}_{3}^{+}+\mathrm{CH}_{4} \rightarrow \mathrm{C}_{2} \mathrm{H}_{5}^{+}+\mathrm{H}_{2}$ or $\mathrm{C}_{2} \mathrm{H}+\mathrm{C}_{2} \mathrm{H}_{2} \rightarrow \mathrm{C}_{4} \mathrm{H}_{2}+\mathrm{H}$ ); and (c) radiative association reactions (e.g. $\mathrm{C}^{+}+\mathrm{C}_{n} \rightarrow \mathrm{C}_{n+1}^{+}+\mathrm{h} \nu$ ). In general, carbon insertion with $\mathrm{C}^{+}$is thought to be the dominant route. Because this leads to loss of at least one hydrogen and because the larger ions $\mathrm{C}_{n} \mathrm{H}_{m}^{+}$do not react rapidly with $\mathrm{H}_{2}$, it is not surprising that this chemistry produces highly unsaturated hydrocarbons, in agreement with the observations of pre-star-forming cores (see Section 6). Reactions with C may be competitive if they are as rapid as suggested by recent laboratory experiments (Kaiser et al 1997; see Smith 1997 for a review).

The buildup of polyatomic hydrocarbons is limited by photodissociation at the edges of clouds or near young stars. This process dominates the removal of most neutral small molecules up to $A_{V} \approx 2$ mag from the radiation source, whereas at larger depths, reactions with atomic $\mathrm{O}$ and $\mathrm{C}^{+}$become the dominant destruction routes. Once the carbon is locked up in the very stable $\mathrm{CO}$ molecule, the formation of more complex hydrocarbons ceases.

The chemistry of sulfur is interesting because all of the gas-phase formation routes to hydrides involve at least one endothermic reaction. Thus, the presence of $\mathrm{H}_{2} \mathrm{~S}$ in cold clouds signifies the importance of other processes, such as grain-surface chemistry. Other sulfur-containing species can be readily produced through ion-molecule and neutral-neutral reactions with carbon- and oxygen-containing species. For example, CS, which is often used as a tracer of dense star-forming regions, results from reactions of $\mathrm{S}^{+}$and $\mathrm{S}$ with $\mathrm{CH}$ and $\mathrm{C}_{2}$, whereas $\mathrm{SO}$ stems from reactions with $\mathrm{OH}$. The subsequent reaction of $\mathrm{SO}$ with $\mathrm{OH}$ is expected to produce copious amounts of $\mathrm{SO}_{2}$. The lack of abundant $\mathrm{SO}_{2}$ in dark clouds, however, indicates an incomplete understanding of the sulfur chemistry and the amount of sulfur depletion in dense clouds (Palumbo et al 1997).

\subsection{Grain-Surface Chemistry}

The chemistry on the surfaces of interstellar grains has received ample discussion in the literature (e.g. Tielens \& Hagen 1982, d'Hendecourt et al 1985, Tielens \& Allamandola 1987, Hasegawa \& Herbst 1993a,b, Herbst 1993, Schutte 1996, Williams \& Taylor 1996). Its main characteristic at low temperatures and densities is the production of hydrogenated species such as $\mathrm{H}_{2} \mathrm{O}, \mathrm{NH}_{3}$, and $\mathrm{CH}_{4}$ owing to the high mobility of atomic hydrogen on the cold surfaces. Hydrogenation of solid $\mathrm{CO}$ can lead to $\mathrm{H}_{2} \mathrm{CO}$ and $\mathrm{CH}_{3} \mathrm{OH}$, although there still is some discussion on the efficiencies of these reactions (Tielens \& Charnley 1997). As the density increases, the amount of gas-phase atomic hydrogen drops precipitously and reactions with atomic oxygen become important, e.g. $\mathrm{CO}+\mathrm{O} \rightarrow \mathrm{CO}_{2}$. Tunneling reactions with $\mathrm{H}_{2}$ occur competitively once $\mathrm{H}_{2} \gg$ $\mathrm{H}$. There is also laboratory evidence that solid-state acid-base reactions (e.g. 
$\mathrm{HCOOH}+\mathrm{NH}_{3} \rightarrow \mathrm{HCOO}^{-}+\mathrm{NH}_{4}^{+}$) can proceed at very low temperatures (Schutte \& Greenberg 1997). At higher temperatures, the diffusion of heavier species such as radicals over the surface becomes significant, allowing a more complex but poorly understood surface chemistry to proceed (e.g. Caselli et al 1993). Above $\sim 60 \mathrm{~K}$, polymerization reactions involving $\mathrm{H}_{2} \mathrm{CO}, \mathrm{NH}_{3}$, and $\mathrm{CH}_{3} \mathrm{OH}$ ice can produce compounds of high molecular weight (Schutte et al 1993).

Photochemical reactions within the ices can be triggered by external ultraviolet photons, by ultraviolet photons from the young star impinging either directly on the inner envelope or scattered by the dust in the outflow into the outer envelope (Spaans et al 1995), or by internal cosmic-ray-produced photons (e.g. Gredel et al 1989). The latter process is always operative and generates an ultraviolet field of $\sim 3 \times 10^{3}$ photons s $\mathrm{sm}^{-1}$ inside dense clouds for a typical cosmic-ray ionization rate of $1.7 \times 10^{-17} \mathrm{~s}^{-1}$ (Cecchi-Pestellini \& Aiello 1992). This is about five orders of magnitude less than the general radiation field at the edge, but it may affect the chemistry on time scales of $\sim 10^{6}$ years. Photodissociation can lead to radicals (e.g. $\mathrm{H}_{2} \mathrm{O} \rightarrow \mathrm{OH}+\mathrm{H}$ or $\mathrm{O}+\mathrm{H}_{2}$ ), which can subsequently react to form other molecules (e.g. $\mathrm{CO}+\mathrm{OH} \rightarrow \mathrm{CO}_{2}$ ) (e.g. Allamandola et al 1988, Grim et al 1989, Bernstein et al 1995, Gerakines et al 1996). In addition, the energy provided by the ultraviolet photons enables new reaction pathways to proceed that differ from those resulting from simple warming because of the presence of radicals. Photolysis of ice mixtures containing $\mathrm{H}_{2} \mathrm{O}, \mathrm{NH}_{3}$, and $\mathrm{CO}$ or $\mathrm{CH}_{3} \mathrm{OH}$ often leaves a nonvolatile organic residue containing a variety of complex oxygen- and nitrogen-rich organic molecules (e.g. d'Hendecourt et al 1982, Briggs et al 1992, Jenniskens et al 1993, Bernstein et al 1995, Greenberg et al 1995). Some of these effects are also found in experiments in which ices are bombarded with highly energetic charged particles, analogous to cosmic rays or X rays (e.g. Moore et al 1983, Strazzula \& Baratta 1992, Kaiser \& Roessler 1997). The thermal heating, photochemical, and irradiation processes are often referred to in the literature as energetic processing, without discrimination.

\section{INVENTORY OF MAJOR O-, C-, AND N-BEARING SPECIES}

The following sections are focused on those molecules whose abundances are most affected by the various phases of star formation. These species are often only minor in terms of overall composition $\left(\sim 10^{-11}-10^{-7}\right.$ with respect to hydrogen), so a brief review of the major reservoirs of $\mathrm{O}, \mathrm{C}$, and $\mathrm{N}$ in dense clouds is useful. Previously, these were discussed with respect to the solar abundances (e.g. van Dishoeck et al 1993), but it has recently been recognized 
that the interstellar abundances of carbon, oxygen, and nitrogen are about 20-30\% lower (e.g. Cardelli et al 1996, Snow \& Witt 1996, Meyer et al 1997; see Meyer 1997 for a review).

A substantial fraction of the refractory material must be in some form of silicate or oxide containing practically $100 \%$ of the interstellar Si and Fe, based on the strong 9.7- and $18-\mu \mathrm{m}$ bands (e.g. Draine \& Lee 1984). With four $\mathrm{O}$ per $\mathrm{Si}$, these silicates account for $\sim 25 \pm 5 \%$ of the oxygen. Carbonaceous materials also are widespread in the solid phase, but their composition is not well known (see Henning 1997 for review). Proposed materials include graphite, hydrogenated amorphous carbon, organic refractory mantles, diamonds, silicon carbide, and solid aromatics, together with large gas-phase molecules such as polycyclic aromatic hydrocarbons, carbon chains, and perhaps fullerenes and fulleranes. Estimates of the abundances are hampered by the lack of firm identifications and laboratory data on band strengths, but together they clearly tie up a large fraction (at least 60\%) of the interstellar carbon (Mathis 1996, Li \& Greenberg 1997). In contrast, at most a few percent of the nitrogen seems to be incorporated in refractory solids.

In the gas phase, most models predict that $\mathrm{O}_{2}$ and $\mathrm{O}$ are the major oxygenbearing species in quiescent cold clouds. Ground-based searches for galactic ${ }^{16} \mathrm{O}^{18} \mathrm{O}$ have resulted in upper limits of $\mathrm{O}_{2} / \mathrm{H}_{2}<10^{-5}$ or $\mathrm{O}_{2} / \mathrm{CO}<0.07$ (Pagani et al 1993, Marechal et al 1997). More sensitive limits of $\mathrm{O}_{2} / \mathrm{CO}<0.01$ have been obtained from emission and absorption line studies of external galaxies (Liszt 1992, Combes \& Wiklind 1995, Combes et al 1997). Thus, gas-phase $\mathrm{O}_{2}$ does not appear to be a major reservoir, although space-borne searches with SWAS (Submillimeter Wave Astronomy Satellite), ODIN [Satellite for Astronomy and Aeronomy at (Sub)millimeter Wavelengths], and FIRST (Far-Infrared and Submillimeter Space Telescope) are needed to settle this issue. Observations of [O I] $63-\mu \mathrm{m}$ absorption suggest that at least $40 \%$ of the oxygen is in atomic form in dense, partly translucent clouds (Poglitsch et al 1996, Baluteau et al 1997). In cold clouds, the gas-phase $\mathrm{H}_{2} \mathrm{O}$ abundance is low, $\sim 10^{-8}-10^{-7}$ (Zmuidzinas et al 1995, Tauber et al 1996), but in warm clouds, a significant fraction of the oxygen is driven into $\mathrm{H}_{2} \mathrm{O}$ (e.g. Cernicharo et al 1997). Gasphase CO contains up to $20 \%$ of the oxygen and up to $40 \%$ of the interstellar carbon. Atomic $\mathrm{C}$ and $\mathrm{C}^{+}$contribute only a few percent inside dense clouds.

Direct observations of the 3- and 6- $\mu \mathrm{m} \mathrm{H}_{2} \mathrm{O}$ ice bands together with the 9.7- $\mu \mathrm{m}$ silicate feature indicate that $5-25 \%$ of the oxygen budget is locked up in water ice in star-forming regions (Whittet 1993), whereas up to $25 \%$ may be in water ice in quiescent cold clouds (Schutte 1996). Solid $\mathrm{O}_{2}$ is very difficult to observe owing to its extremely weak infrared bands (Ehrenfreund et al 1992), but sensitive searches with ISO give an upper limit of $25 \%$ with respect to $\mathrm{H}_{2} \mathrm{O}$-ice for one line of sight (B Vandenbussche, P Ehrenfreund, ACA Boogert, 
PA Gerakines, EF van Dishoeck, et al, in preparation). Solid $\mathrm{CO}$ and $\mathrm{CO}_{2}$ account for less than $10 \%$ of the total oxygen toward YSOs (Whittet et al 1996), whereas they contain $\sim 13 \%$ in quiescent clouds (Whittet et al 1998).

For nitrogen, models predict that $\mathrm{N}$ and $\mathrm{N}_{2}$ dominate the gas-phase chemistry, but both are undetectable in dense clouds. Some indirect limits on the $\mathrm{N}_{2}$ abundance are available from observations of the $\mathrm{N}_{2} \mathrm{H}^{+}$ion in large beams, which indicate that $\mathrm{N}_{2}$ contains on average only $\sim 10 \%$ of the available nitrogen (McGonagle et al 1990, Womack et al 1992). However, in some dense starforming cloud cores like NGC 2264 IRS1, the fraction may be increased to nearly 100\% (van Dishoeck et al 1992, de Boisanger et al 1996). A recent $\mathrm{N}_{2} \mathrm{H}^{+}$survey of a set of embedded YSOs in Taurus and Serpens also suggests $\mathrm{N}_{2}$ fractions of 30-100\% (C Qi, GA Blake, EF van Dishoeck, P Bergman, in preparation). The remainder is probably in the form of gas-phase $\mathrm{N}$, although the amount of solid $\mathrm{N}_{2}$ is unknown. The amount of solid $\mathrm{NH}_{3}$ is only a few percent of that of $\mathrm{H}_{2} \mathrm{O}$ ice.

In summary, most of the oxygen seems to be accounted for in silicates, ices, and gas-phase $\mathrm{O}, \mathrm{CO}$, and possibly $\mathrm{H}_{2} \mathrm{O}$ if the lower interstellar abundances are adopted. Most of the carbon is in some solid carbonaceous form, with the remainder in gas-phase CO. Nitrogen is mostly locked up in gas-phase $\mathrm{N}_{2}$ and $\mathrm{N}$, although the amount in solids is poorly constrained.

\section{CHEMICAL MODELS}

\subsection{Gas-Phase Models}

The calculation of chemical abundances in dense clouds and YSOs requires a specification of the physical parameters such as the temperature, density, and radiation field. In their simplest form, two different classes of models are considered: 1. Steady-state, depth-dependent models, in which the abundances of the molecules do not change with time but are functions of depth into the region. Models of the translucent outer envelopes (e.g. van Dishoeck 1998a) and dense ultraviolet photon- or X-ray-dominated regions near young stars (Hollenbach \& Tielens 1997, Sternberg et al 1997) fall in this category, but they are generally not applied to the bulk of star-forming regions. 2. Time-dependent, depthindependent models, in which the concentrations are computed as functions of time at a single position deep inside the cloud. Models of dark pre-star-forming clouds (e.g. Millar et al 1991b, 1997a), collapsing envelopes (e.g. Bergin \& Langer 1997), and hot cores near massive young stars (e.g. Charnley et al 1992) fall in this category. The time scale for reaching chemical equilibrium ranges from $10^{5}$ to $10^{7}$ years, depending on the degree of ionization, temperature, density, and the species involved. 
Several parameters enter the models. In both cases, $(a)$ the elemental abundances of $\mathrm{C}, \mathrm{O}, \mathrm{N}, \mathrm{S}$, metals, etc and $(b)$ the primary cosmic-ray ionization rate $\zeta_{o}$ need to be specified. For steady-state, depth-dependent models, additional parameters are $(c)$ the geometry (e.g. plane-parallel, spherical, etc); $(d)$ the density $n_{\mathrm{H}}=n(\mathrm{H})+2 n\left(\mathrm{H}_{2}\right)$ as a function of position; $(e)$ the incident radiation field; and $(f)$ the grain parameters, i.e. the extinction curve, albedo, and scattering function. The temperatures of the gas and dust as functions of position into the region can be obtained self-consistently from the balance of heating and cooling processes. Alternatively, they can be constrained from observations and provided as additional input parameters.

In the time-dependent models, the parameters besides $(a)$ and $(b)$ are the density as a function of time; the visual extinction $A_{V}$ at the position in the cloud, usually taken to be so large that external photochemistry can be neglected; and the initial abundances of the various species at $t=0$, usually taken to be atomic except for $\mathrm{H}_{2}$. Again, the temperature can be obtained from the thermal balance but is often set at $10 \mathrm{~K}$ for both the gas and dust, typical of a dark cloud shielded from ultraviolet radiation and heated by cosmic rays only. In these models, the ratios of the local concentrations are taken to be equal to the ratios of the column densities integrated over depth. This procedure is valid for molecules whose abundances peak in the center of the core or cloud, but it may lead to incorrect results for species such as radicals whose abundances peak in the outer part of the envelope. Another complication is that the timedependent models have more than one steady-state solution in certain regions of parameter space (e.g. Le Bourlot et al 1995), although it is not yet clear what the astrophysical consequences of this "bistability" phenomenon are (e.g. Shalabiea \& Greenberg 1995a).

The principal chemical characteristics of both the depth- and time-dependent models are governed by the transition of carbon from atomic to molecular form. In the depth-dependent case, $\mathrm{C}^{+}$recombines to $\mathrm{C}$ around $A_{V} \approx 1 \mathrm{mag}$, followed by the conversion to $\mathrm{CO}$ near $A_{V} \approx 2 \mathrm{mag}$. The $\mathrm{CO}$ photodissociation rate as a function of depth is crucial in this transition, and its calculation requires a careful treatment of the complicated radiative transfer (van Dishoeck \& Black 1988, Warin et al 1996a, Lee et al 1996b). In the time-dependent models, the same chemical characteristics are seen if depth is replaced by time and if all species except $\mathrm{H}_{2}$ are initially in atomic form, with carbon present as $\mathrm{C}^{+}$. On a time scale of $\sim 10^{3}$ years, $\mathrm{C}^{+}$recombines to $\mathrm{C}$, which subsequently transforms to $\mathrm{CO}$ after $\sim 10^{5}$ years. Since the presence of atomic carbon is essential to building up more complex organic molecules and long carbon chains such as $\mathrm{C}_{2} \mathrm{~S}$ and $\mathrm{HC}_{3} \mathrm{~N}$, these species are abundant only at early times and drop as a steady state is approached. 
In the "pseudo"-time-dependent models, the density is taken to be constant with time. A proper theoretical treatment of the chemistry in star-forming regions requires the coupling of the chemical models with multidimensional hydrodynamical codes for all phases of the star-formation process, including the collapse phase, the disk formation epoch, the development of the bipolar outflow, and the dispersal of the envelope (e.g. Boss \& Myhill 1995, Yorke et al 1995). The coupling of the processes at each point of the hydrodynamical flow is important, since the chemical composition determines the cooling rates and since the dynamical and chemical time scales are comparable. Such complete chemical-hydrodynamical codes are not yet feasible computationally, so current models make simplifications on either the chemical or the dynamical side. Early dynamical models with limited chemistry include those of Gerola \& Glassgold (1978), Tarafdar et al (1985), Umebayashi \& Nakano (1990), and Prasad et al (1991). Examples of more recent work are by Nelson \& Langer (1997) and Shematovich et al (1997).

More relevant for this review are models that couple a detailed chemical network with parametrized fits to the density profiles resulting from simple dynamical models such as that of Shu (1977) and Basu \& Mouschovias (1994). Models in this category include those of Rawlings et al (1992), Shalabiea \& Greenberg (1995b), and Ceccarelli et al (1996), who take full account of the changing physical conditions at every point in the collapsing envelope at all times. Bergin \& Langer (1997) considered the evolution of a single parcel of gas deep inside the core.

A different class of pseudo-time-dependent models relevant to star formation are those appropriate for hot cores, in which the temperatures are much higher and the initial composition of the gas is not taken to be atomic but molecular, such that it is consistent with the composition of evaporating ice mantles in the vicinity of young stars. This results in a rapid gas-phase chemistry at high temperatures in which copious amounts of complex saturated organics are produced (Charnley et al 1992, Caselli et al 1993, Millar 1997; see Section 9).

YSOs are known to be strong emitters of X rays (e.g. Casanova et al 1995), which may affect the physical and chemical structure of the immediate circumstellar environments. For modest enhancements of the ionization rate (a factor of $\sim 1000$ or less) compared with the standard cosmic-ray rate, the formation of molecules is accelerated (Krolik \& Kallmann 1983) and species such as OH, $\mathrm{H}_{2} \mathrm{O}$, and $\mathrm{HCO}^{+}$are produced in abundance. However, for higher ionization rates, $\mathrm{H}_{2}$ is destroyed, along with most other molecules (Lepp \& Dalgarno 1996). Detailed models of such X-ray dissociation regions (XDRs) have been presented by Maloney et al (1996) and Yan \& Dalgarno (1997), whereas the effects of $\mathrm{X}$ rays on the ionization structure of circumstellar disks have been considered by Glassgold et al (1997) and Shu et al (1997). 


\subsection{Gas-Grain Models}

The coupling of gas and grain chemistry has been incorporated in chemical models through two different approaches (Tielens \& Charnley 1997, Tielens \& Whittet 1997). In the "accretion-limited" regime, the time scale for a mobile species to scan the surface is much less than the accretion time of the coreactant. The chemistry is then limited by the accretion rate of new species. In the "reaction-limited" regime, the opposite holds true, so a species trapped in a site can react only with migrating species that visit that site. Most of the chemical models incorporating gas-grain interactions have been formulated in the reaction-limited regime through the use of rate equations for computational convenience (e.g. Hasegawa \& Herbst 1993a,b, Shalabiea \& Greenberg 1995b). However, under dark cloud conditions, the surface chemistry is likely to be in the accretion-limited regime and can only be properly treated by a Monte Carlo method (e.g. Tielens \& Hagen 1982). Recently, Caselli et al (1998a) have attempted to modify the rate equations to take into account the shortcomings of the reaction-limited approach. The effects on the published model results for star-forming regions remain to be assessed.

Another essential ingredient of the gas-grain models is the mechanism for returning the molecules to the gas phase. If no desorption is included, the gasphase molecules accrete onto grain surfaces on a time scale of $\sim 2 \times 10^{9} / n_{\mathrm{H}} y_{S}$ year, where the sticking coefficient $y_{S}$ is thought to lie between 0.1 and 1.0 (Williams 1993). Thus, for typical dark cloud densities of $10^{4} \mathrm{~cm}^{-3}$, most molecules should disappear from the gas phase in less than $10^{6}$ years. Since this is inconsistent with the observed widespread molecular emission, it implies the presence of efficient desorption mechanisms, even in the coldest, most quiescent clouds. Possible mechanisms and their time scales have been summarized by Schutte \& Greenberg (1991), Williams (1993), Schmitt (1994), and Schutte (1996). Thermal evaporation is effective only at higher temperatures once the star has formed, $T_{\mathrm{d}} \gtrsim 20 \mathrm{~K}$. The energy liberated by the formation of molecules heats the grains locally and may remove some species (Willacy et al 1994b). Other effective desorption mechanisms in cold gas are thought to be cosmicray spot heating (Léger et al 1985) and explosive heating due to exothermic reactions between radicals (d'Hendecourt et al 1982), which can be triggered either by cosmic rays or by grain-grain collisions at velocities greater than $\sim 0.1 \mathrm{~km} \mathrm{~s}^{-1}$. The efficiencies of all these mechanisms depend strongly on the binding energies of the molecules on the surfaces, which are different for bare silicates, $\mathrm{H}_{2} \mathrm{O}$-rich and CO-rich ice mantles (Hasegawa \& Herbst 1993a). Polar molecules such as $\mathrm{H}_{2} \mathrm{O}$, which contain strong hydrogen bonds in the ices, are difficult to remove from any surface in cold clouds, whereas nonpolar species such as $\mathrm{N}_{2}$ have very low binding energies and are easily returned to 
the gas phase. Additional laboratory experiments are needed to determine the efficiency of these processes.

\section{CHEMISTRY IN PRESTELLAR CORES}

\subsection{Low-Mass Cores}

The chemical evolution of star-forming regions starts with the dark and dense clouds out of which the new stars are assembled. Most observational and theoretical studies of dark cloud chemistry have focused on TMC-1 and L134N, clouds that may eventually form low-mass stars. TMC- 1 shows a particularly rich chemistry with large chemical gradients over the 0.6-pc long ridge and high abundances of unsaturated carbon chain molecules in the southern region (e.g. Hirahara et al 1992, Ohishi et al 1992, Pratap et al 1997). Pseudo-timedependent gas-phase models can fit the abundances of the carbon chains only at early times of $t \sim 10^{5}$ years (e.g. Lee et al 1996a,b, Millar et al 1997a, Pratap et al 1997; but see Ruffle et al 1997 for an alternative explanation). It should be kept in mind, however, that other mechanisms that bring fresh atomic carbon inside a molecular cloud, such as turbulent mixing (Xie et al 1995), may give similar results, such that the inferred "chemical" age does not necessarily reflect the true physical age. Howe et al (1996) have recently modeled the chemical gradient across TMC-1 within the dynamical framework of sequential fragmentation along the ridge presented by Hanawa et al (1994). They concluded that TMC-1 may be chemically rich compared with other clouds because it is undergoing its first cycle of (sudden) collapse starting from very diffuse, atomic-carbon-rich gas. On the other hand, Pratap et al (1997) showed that a small change in the gas-phase $\mathrm{C} / \mathrm{O}$ abundance ratio caused, for example, by a slight density change can explain the observed gradient.

To obtain further insight into the nature of pre-star-forming cores, Suzuki et al (1992) performed a systematic study of a few characteristic molecules $\left(\mathrm{C}_{2} \mathrm{~S}\right.$, $\mathrm{HC}_{3} \mathrm{~N}, \mathrm{HC}_{5} \mathrm{~N}$, and $\mathrm{NH}_{3}$ ) in a set of dark cores identified by Myers \& Benson (1983). The abundances of the carbon chain molecules correlate well with each other but not with $\mathrm{NH}_{3}$, which was found to be more abundant in older cores where stars have already formed. The observed $\mathrm{C}_{2} \mathrm{~S} / \mathrm{NH}_{3}$ abundances can be reproduced quantitatively in models that start from diffuse gas and form dense cores over a period of $10^{5}$ to $2 \times 10^{6}$ years. Thus, this ratio may be a particularly useful tracer of cloud evolution if the proposed scenario is valid. This point is illustrated in more detail by observations of the quiescent core L1498 by Kuiper et al (1996) and Wolkovitch et al (1997). High-resolution data show a chemically differentiated onion-shell structure, with $\mathrm{NH}_{3}$ peaking in the inner and $\mathrm{C}_{2} \mathrm{~S}$ in the (clumpy) outer parts (see Figure 3). The core is thought to be roughly in gravitational equilibrium but still growing in mass owing to accretion of less dense, atomic-carbon-rich halo material. 


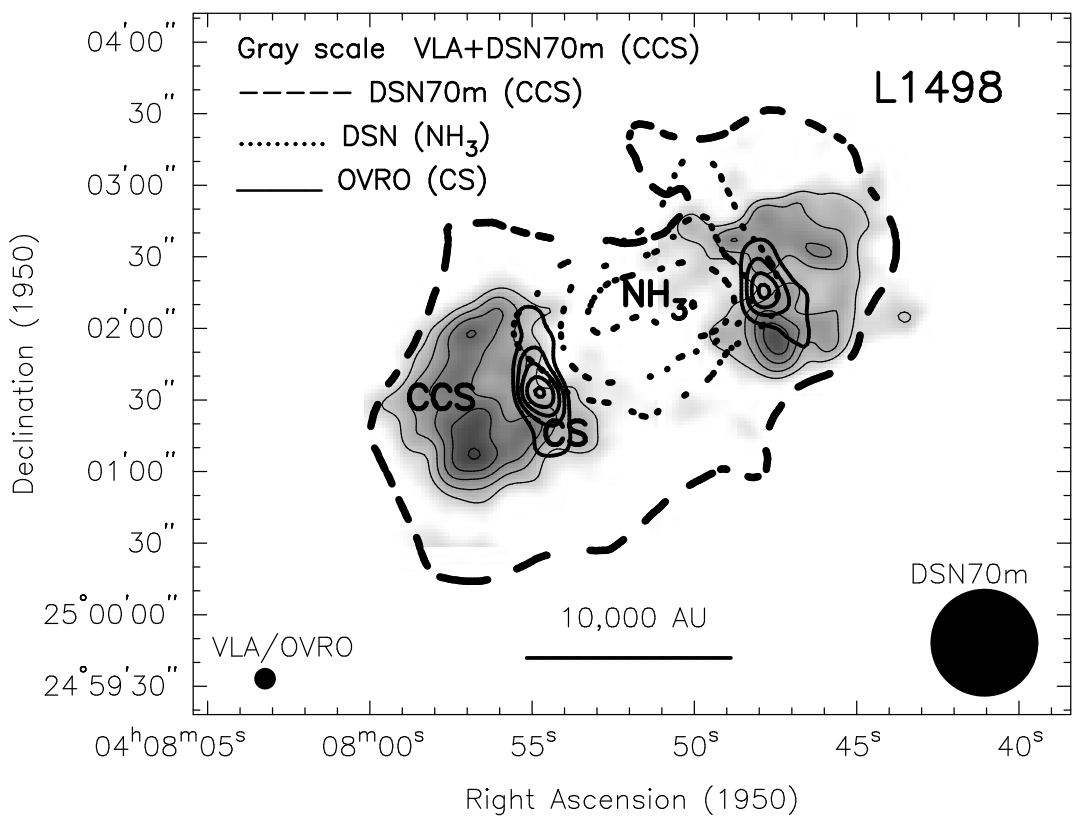

Figure $3 \mathrm{C}_{2} \mathrm{~S}, \mathrm{CS}$, and $\mathrm{NH}_{3}$ observations of the prestellar core L1498, showing a chemically differentiated structure with unsaturated carbon chain molecules such as $\mathrm{C}_{2} \mathrm{~S}$ that are more abundant in the atomic-carbon-rich outer part and $\mathrm{NH}_{3}$ peaking in the inner region (Kuiper et al 1996).

Prestellar cores such as TMC-1 and L1498 have a relatively flat density distribution on scales of $0.02-0.05 \mathrm{pc}$. Recently, a set of centrally concentrated prestellar cores has been discovered by submillimeter continuum observations of the Taurus and Ophiuchus regions (e.g. Ward-Thompson et al 1994, Motte et al 1998). Myers et al (1996) also noted that L1544 may be an evolved prestellar core on the verge of collapse. Systematic chemical studies of these objects have yet to be performed and would be very interesting, since they have a well-defined density structure and are likely to be the immediate precursors to protostellar objects.

Ice mantles have been observed in such quiescent dark clouds through observations of field stars in Taurus and Serpens with up to 20 mag of extinction (e.g. Chiar et al 1995, Whittet et al 1998). $\mathrm{H}_{2} \mathrm{O}, \mathrm{CO}$, and $\mathrm{CO}_{2}$ are clearly detected at abundances that indicate that up to $40 \%$ of the heavy elements may be frozen out at densities of a few times $10^{4} \mathrm{~cm}^{-3}$ (see Section 4). The amount of solid $\mathrm{CO}$ is comparable to that of gas-phase $\mathrm{CO}$, and the solid $\mathrm{CO}$ profile shows a large nonpolar component with no evidence for processing or heating above $10 \mathrm{~K}$. 


\subsection{High-Mass Giant Molecular Cloud Cores}

Analyses of the chemical composition of Giant Molecular Clouds (GMCs) that form high-mass stars have been performed by Bergin et al (1997b) and Ungerechts et al (1997), who have systematically mapped several GMC cores (Orion, M17, and Cepheus A) over a $5^{\prime} \times 5^{\prime}$ region in various molecules. The chemical composition was analyzed at several positions away from the YSOs. The derived abundances are remarkably uniform (within a factor of a few), both within a single GMC and between different GMCs, in contrast with the situation for some dark cores. Bergin et al (1997a) ran several gas-phase and gas-grain models for comparison. Pure gas-phase models are most successful in reproducing the observed abundances at early times $\left(\sim 10^{5}\right.$ years $)$ if a fairly high gas-phase elemental abundance ratio $[\mathrm{C}]_{\mathrm{g}} /[\mathrm{O}]_{\mathrm{g}} \approx 0.8$ is adopted. They argue that the observed CS/SO ratio may be used to constrain this ratio, as has also been suggested for the L134N and TMC-1 dark cores (Swade 1989, Pratap et al 1997). The $\mathrm{HNC} / \mathrm{HCN}$ ratio is found to be particularly sensitive to temperature (e.g. Schilke et al 1992).

\subsection{Ionization Fraction}

An important parameter for the dynamical evolution of star-forming regions is the ionization fraction, $x(\mathrm{e})=n(\mathrm{e}) / n\left(\mathrm{H}_{2}\right)$, since the charged species govern the coupling of the gas to magnetic fields and therefore the ability of a cloud to collapse and form stars. In the model calculations, the electron abundance drops from its high value of $\sim 10^{-4}$ at the edge to a few times $10^{-9}-10^{-8}$ in the center of a dense core. $\mathrm{H}_{3}^{+}, \mathrm{HCO}^{+}$, and $\mathrm{H}_{3} \mathrm{O}^{+}$are expected to be the principal molecular ions, in addition to metal ions such as $\mathrm{Mg}^{+}$and $\mathrm{Fe}^{+}$. The predicted electron abundance scales with $\left(\zeta / n_{\mathrm{H}}\right)^{1 / 2}$ if the metal abundance is low and $\left(\zeta / n_{\mathrm{H}}\right)^{1 / 3}$ if metals are not taken to be significantly depleted (Oppenheimer \& Dalgarno 1974, Millar 1990). Simplified expressions can be found in Basu \& Mouschovias (1994) and McKee (1989), whereas detailed fits to model calculations of $x(\mathrm{e})$ in collapsing envelopes have been given by Bergin \& Langer (1997).

Observationally, the traditional method to constrain $x(\mathrm{e})$ involves a determination of the $\mathrm{DCO}^{+} / \mathrm{HCO}^{+}$ratio (Langer 1985, Caselli et al 1998b). de Boisanger et al (1996) extended this method to observations of several protonated molecular ions to constrain both $x(\mathrm{e})$ and $\zeta_{o}$ in two dense clouds. The observed abundances of the positive ions provide a lower limit of $x(\mathrm{e}) \gtrsim 3 \times$ $10^{-9}$, whereas the estimated ionization fractions range from $\sim 10^{-8}$ to a few times $10^{-7}$ for various clouds (Schilke et al 1991, de Boisanger et al 1996, Caselli et al 1998b), with inferred values of $\zeta_{o}$ between $10^{-17}$ and a few times $10^{-16} \mathrm{~s}^{-1}$. No systematic trends between pre-star-forming cores and cores with stars have yet been found, but this may be due to uncertainties in the analysis of the sample obtained to date. 


\section{CHEMISTRY IN THE COLD COLLAPSE ENVELOPES AROUND DEEPLY EMBEDDED YOUNG STELLAR OBJECTS}

\subsection{Models of Envelopes Around Low-Mass Young Stellar Objects}

Once collapse occurs, the increasing density and decreasing temperature accelerates the gas-phase depletion of molecules onto grain mantles, while the radiative heating and outflow from the central star act to return molecules into the gas phase. In the models of Rawlings et al (1992), Willacy et al (1994a), Bergin \& Langer (1997), and Shalabiea \& Greenberg (1995b), no heating from the protostar is taken into account, so they are appropriate for the outer region of the collapsing envelope $\left(>10^{16} \mathrm{~cm}\right)$ where the temperature remains low owing to the efficient cooling by the molecules, about $10 \mathrm{~K}$. All four models include depletion onto grains, but they differ strongly in the adopted processes that return molecules to the gas, ranging from none in Rawlings et al's to efficient, explosive desorption in Shalabiea \& Greenberg's. The results also depend on the initial conditions and the adopted binding energies within the icy mantles, especially whether the outer layer is $\mathrm{H}_{2} \mathrm{O}$ rich or $\mathrm{CO}$ rich. If only thermal desorption is taken into account, virtually all species condense from the gas phase onto the grains at $t>10^{6}$ years since the onset of collapse, except $\mathrm{H}_{2}$ and perhaps $\mathrm{N}_{2} . \mathrm{N}_{2} \mathrm{H}^{+}$and $\mathrm{HCO}^{+}$may remain high because the $\mathrm{H}_{3}^{+}$abundance increases when its main removal partners $\left(\mathrm{CO}, \mathrm{O}, \mathrm{H}_{2} \mathrm{O}\right.$, etc) are condensed onto grains. Thus, these ions are predicted to be good tracers of the collapsing envelopes.

Ceccarelli et al (1996) also considered the chemistry and resulting line emission within the inside-out collapse model but down to much smaller scales of $\sim 10$ AU. In addition, their model is distinguished by an explicit treatment of the thermal balance, including heating due to the accretion luminosity of the protostar. Much higher dust temperatures, $T_{\mathrm{d}} \approx 100(r / 100 \mathrm{AU})^{-0.4} \mathrm{~K}$, are found in the inner regions of these models. The gas is heated by collisions with the warm dust, by compressional heating, and by absorption of infrared photons followed by collisional de-excitation, resulting in $T_{\text {gas }} \approx T_{\mathrm{d}}$. This implies that within $r<10^{15} \mathrm{~cm}(<60 \mathrm{AU})$ of the young star, the infalling dust is heated to $100 \mathrm{~K}$, resulting in the sublimation of $\mathrm{H}_{2} \mathrm{O}$-rich ices into the gas. In addition, once the gas is heated to $200-300 \mathrm{~K}\left(r<3 \times 10^{14} \mathrm{~cm}\right)$, the gas-phase chemical reactions convert $\mathrm{O}$ and $\mathrm{O}_{2}$ into $\mathrm{H}_{2} \mathrm{O}$, resulting in high abundances of $\mathrm{H}_{2} \mathrm{O}$ in the inner 10-100 AU. This significantly changes the chemistry in the inner part of the envelope, but little information on species other than $\mathrm{H}_{2} \mathrm{O}, \mathrm{OH}$, and $\mathrm{O}$ is available. It will be interesting to explore the chemistry of other elemental families in such models. 
In the earliest stages, the formation of the new star is accompanied by a powerful bipolar outflow, which creates shocks when it runs into the surrounding envelope, resulting in a characteristic chemistry discussed in more detail in Section 8. The bulk of the envelope mass is not directly influenced by the outflow at this stage, so abundances derived from optically thin single-dish emission well removed from the outflow axis may not be greatly affected. The envelopes can, however, be heated by ultraviolet photons generated in the inner boundary layer between the fast rotating accretion disk and the much slower rotating protostar (e.g. Hartigan et al 1991). Ultraviolet photons can escape through the biconical cavity evacuated by the outflow and be scattered by dust grains into the envelope (Spaans et al 1995). For a typical color temperature of the radiation field of $10,000 \mathrm{~K}$, the photons are not energetic enough to photodissociate $\mathrm{H}_{2}$ and $\mathrm{CO}$ and start an active gas-phase chemistry, but they can heat part of the envelope to $\sim 100 \mathrm{~K}$ and alter the ices.

\subsection{Observations of Envelopes Around Low-Mass Young Stellar Objects}

The principal prediction of collapse models is that most molecules freeze out onto the grains at low temperatures and high densities. This is difficult to prove observationally, however, since every collapsing core has an outer "skin" in which the abundances are normal. Even if this skin amounts to only a few percent of the total column density, the overlying layer in which the abundances are a factor of 10-100 higher can effectively mask any depletions deep inside (Mundy \& McMullin 1997). The most direct method would be to observe the ice mantles through infrared absorption, but at the earliest, deeply embedded stages, the dust obscuration is so high that the sources are not visible even at mid-infrared wavelengths. Careful modeling of the line and dust continuum data appears to be the only way to probe the abundances in this phase, which has indeed resulted in estimated depletions of factors of several to 10 in the inner envelopes of low- and intermediate-mass objects in Serpens and IRAS 05338-0624 (e.g. McMullin et al 1994a,b) to more than 20 in NGC 1333 IRAS4 (Blake et al 1995).

Line profiles are also powerful indicators of the physical and chemical processes taking place in the collapsing envelope (Rawlings et al 1992). Zhou et al (1993), Choi et al (1995), Gregersen et al (1997), and Mardones et al (1997) have used systematic observations of $\mathrm{HCO}^{+}$, CS, and $\mathrm{H}_{2} \mathrm{CO}$ to search for evidence of infall in the earliest stages, including objects such as B335. The choice of molecule is dictated by the requirement that its abundance stays high throughout the envelope, by the optical depth of the lines, and by whether their critical density is appropriate for the infalling material. The low-lying 
lines of the above molecules seem to be the most useful, although the lack of understanding of the sulfur chemistry and depletion casts some doubt on the use of CS (Hartquist et al 1998). The observed narrow $\mathrm{NH}_{3}$ profiles suggest depletion of this species in the inner densest part (Rawlings et al 1992).

A systematic study of the $\mathrm{HCO}^{+}$and/or CS lines in the envelopes of a welldefined set of more evolved embedded objects in Taurus has been performed by Moriarty-Schieven et al (1995), Ohashi et al (1991, 1996), and Hogerheijde et al (1997a, 1998) using a combination of single-dish and interferometer data. These studies confirm that $\mathrm{HCO}^{+}$is an excellent tracer of the envelope structure and mass and that its abundance does not decrease in the innermost regions. In some sources, the $\mathrm{HCO}^{+}$emission appears to be influenced by the outflow, but a more reliable determination of the abundances and possible gradients requires detailed physical models of such objects, coupled with radiative transfer codes that take the velocity structure and outflow cavity into account (MR Hogerheijde \& $\mathrm{F}$ van der Tak, in preparation). $\mathrm{N}_{2} \mathrm{H}^{+}$, on the other hand, seems to trace preferentially the quiescent outer envelope gas in low-mass YSOs (Bachiller 1996, Mardones et al 1997; C Qi, GA Blake, EF van Dishoeck, P Bergman, in preparation). This ion may be destroyed by proton transfer to $\mathrm{CO}$ in dense regions where $\mathrm{CO}$ is not significantly condensed onto the grains. Observations of other molecules at this stage are hampered by the weakness of the lines. For example, Kelly et al (1996) have studied the chemistry toward B5 IRS1 but found low abundances for most species. High-resolution studies by Fuller et al (1991) and Langer et al (1996) show that $\mathrm{HCO}^{+}$traces the envelope and extended circumstellar disk, whereas $\mathrm{HCN}$ seems to avoid the inner part.

At a more evolved but still embedded stage, it becomes possible to probe the ice mantles surrounding such objects (e.g. L1489, Elias 18, R CrA IRS2; Teixeira et al 1998, Chiar et al 1998). Although the near-infrared source is often reflected light off the outflow cavity, it still passes through a sufficiently dense part of the envelope to reveal clear differences with the observations toward field stars. In general, the amount of solid $\mathrm{CO}$ with respect to $\mathrm{H}_{2} \mathrm{O}$ is lower in the YSOs because of some outgassing, on the order of 5-20\% compared with $25-60 \%$ in quiescent clouds, and the $\mathrm{CO}$ profile is broader, indicative of energetic processing. Also, the "XCN" band at $4.62 \mu \mathrm{m}$, thought to be a sign of processing (Section 7.4), has been detected toward a few low-mass YSOs (Tegler et al 1995).

As the objects evolve to the optically visible $\mathrm{T}$ Tauri stage, a larger fraction of the envelope gas and dust is dispersed, making it even more difficult to study its chemistry. However, a circumstellar disk remains, which can be observed now without confusion from the surrounding envelope (see Section 11). 


\subsection{Models of Envelopes of High-Mass Young Stellar Objects}

Self-consistent models of the thermal balance, chemistry, and radiative transfer at all radii $r$ within a spherical envelope have been performed most recently by Doty \& Neufeld (1997). These models extend the work of Ceccarelli et al (1996) to high-mass YSOs with luminosities up to $10^{5} L_{\odot}$, although they do not include the time dependence of the physics and chemistry nor the compressional heating of the gas. Assuming a $r^{-2}$ density distribution, the radial dust and gas temperature profiles and the chemical abundances are computed. Other calculations of the radial dust temperature distribution of high-mass YSOs include those of Campbell et al (1995), Kaufman et al (1998), and van der Tak et al (F van der Tak, EF van Dishoeck, NJ Evans, GA Blake, in preparation). Compared with low-mass YSOs, the temperature is higher over a larger fraction of the envelope, and the cores may also be heated externally by nearby young stars. The region where $T_{\mathrm{d}}>90 \mathrm{~K}$ is increased to $\sim 10^{16} \mathrm{~cm}$ for a $10^{5}-L_{\odot}$ source, resulting in evaporation of $\mathrm{H}_{2} \mathrm{O}$ ice and an enhancement of the gas-phase water abundance by a factor of 100-1000. The chemistry in this inner hot core region is discussed in Section 9.

The temperature and density profiles in collapsing envelopes naturally lead to different ice mantle compositions and infrared spectra compared with those found in uniform, quiescent gas (cf Figure 2; Tielens 1989, Schutte 1996, Tielens \& Whittet 1997). First, the density profile results in a steep gradient in the gas-phase H/CO ratio: At the lower densities $\left(<5 \times 10^{3} \mathrm{~cm}^{-3}\right)$, the atomic hydrogen abundance in the gas is high and most of the heavy elements accrete as atoms, leading to polar $\mathrm{H}_{2} \mathrm{O}, \mathrm{CH}_{4}$, and $\mathrm{NH}_{3}$ ices. At intermediate densities $\left(10^{4}-10^{5} \mathrm{~cm}^{-3}\right)$, most of the gas phase carbon is tied up as CO. The accreted $\mathrm{CO}$ can react with the small amount of atomic hydrogen maintained by cosmicray-induced processes to produce $\mathrm{H}_{2} \mathrm{CO}$ and $\mathrm{CH}_{3} \mathrm{OH}$ and with atomic $\mathrm{O}$ to give $\mathrm{CO}_{2}$. At high densities $\left(>10^{5} \mathrm{~cm}^{-3}\right)$, most of the oxygen and nitrogen are in $\mathrm{O}_{2}$ and $\mathrm{N}_{2}$ in the gas, resulting in nonpolar ices consisting of accreted $\mathrm{CO}, \mathrm{O}_{2}$, and $\mathrm{N}_{2}$, with some $\mathrm{CO}_{2}$ and $\mathrm{H}_{2} \mathrm{O}$ formed through an $\mathrm{H}_{2} \mathrm{O}_{2}$ route. Because the density profile changes with time, layered ices can be produced in a collapsing envelope, with the polar ices condensing first and the nonpolar species forming a volatile "crust."

Second, desorption processes can also shape the composition of the ice mantles (see Figure 4). This is caused by the fact that virtually all desorption mechanisms are much more efficient for volatile species $\left(\mathrm{CO}, \mathrm{O}_{2}, \mathrm{~N}_{2}\right)$ than for nonvolatile material $\left(\mathrm{H}_{2} \mathrm{O}, \mathrm{CH}_{3} \mathrm{OH}\right)$. This "distillation" effect thus decreases the nonpolar ices compared with the polar ices around protostars. Specifically, the 


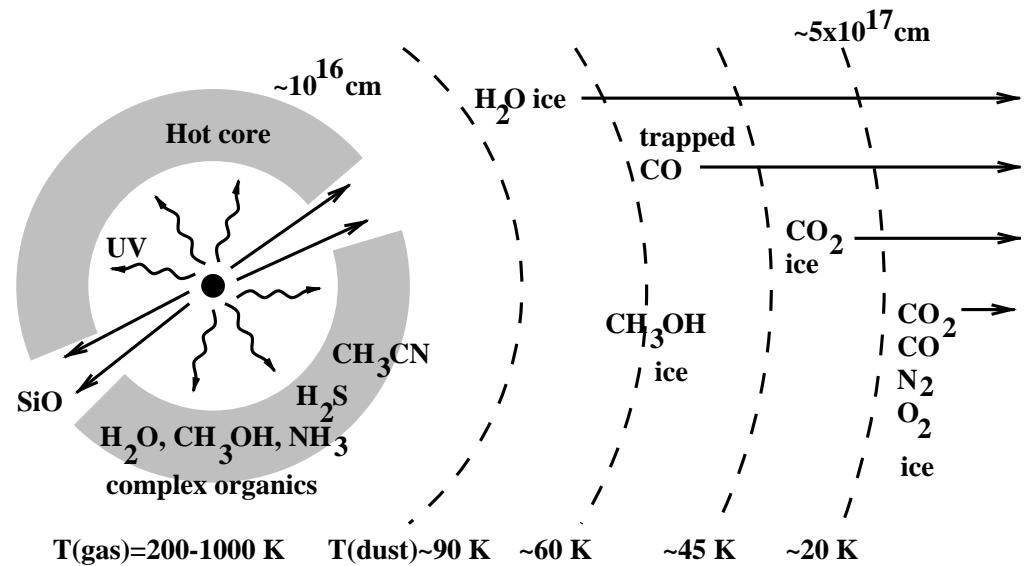

Figure 4 Schematic illustration of the chemical environment of massive YSOs. The variation in the chemical structure of the ice mantle in the envelope due to thermal desorption is shown (based on Tielens et al 1991, Williams 1993).

temperature gradient in the envelope results in evaporation of the volatile ice components $\left(\mathrm{CO}, \mathrm{CO}_{2}\right)$ in the inner part at temperatures above $\sim 20$ and $\sim 45 \mathrm{~K}$, respectively. Molecules in mixed polar ices are expected to evaporate around the same temperature as $\mathrm{H}_{2} \mathrm{O}, \sim 90 \mathrm{~K}$ (Sandford \& Allamandola 1993). Heating of ices may cause rearrangement of the ice matrix, resulting in segregation or expulsion of species like $\mathrm{CH}_{3} \mathrm{OH}$ and $\mathrm{CO}_{2}$ from the mantle into separate phases at temperatures controlled by phase changes in the $\mathrm{H}_{2} \mathrm{O}$-rich ices (Blake et al 1991). A third possibility for ice segregation is driven by the grain-size distribution, since the smallest grains experience temporal temperature excursions due to absorption of an ultraviolet photon, which may lead to evaporation of volatiles. Finally, ultraviolet and $\mathrm{X}$ rays from the young stars can process these layered ices to more complex nonvolatile organics (Greenberg et al 1993, 1995, Pendleton et al 1994). Cycling of the dust grains through the diffuse and dense phases of the interstellar medium can result in a mix of all these effects.

\subsection{Observations of Envelopes Around High-Mass Young Stellar Objects}

A large range of observational data of various molecular lines toward massive YSOs is scattered throughout the literature. Such data were taken for a variety of purposes, ranging from determination of the physical parameters using $\mathrm{H}_{2} \mathrm{CO}$ 
(e.g. Mangum \& Wootten 1993) or CS (e.g. Plume et al 1997) to chemical studies of selected minor species like NS (McGonagle \& Irvine 1997) or $\mathrm{SO}^{+}$ (Turner 1994). Compared with low-mass objects, the lines are much stronger, so the chemistry of minor species can be probed. Systematic studies of the chemistry using a homogeneous set of data on high-excitation lines in small beams $\left(\leq 20^{\prime \prime}\right)$ have been performed for only a handful of objects, including W3 IRS5 (Helmich \& van Dishoeck 1997), NGC 2264 IRS1 (Schreyer et al 1997), AFGL 2591 (F van der Tak, EF van Dishoeck, NJ Evans, GA Blake, in preparation), and AFGL 2136 (AMS Boonman, F van der Tak, FP Helmich, EF van Dishoeck, in preparation). Variations between the abundances of various molecules are seen, especially of species containing nitrogen and sulfur, but no detailed models have been made yet. As for low-mass objects, accurate determination of the abundances requires the availability of a detailed physical model of the region in order to disentangle the contributions from the various physical components and constrain possible radial abundance gradients.

There has been a long-standing debate as to whether substantial depletion is observed in the massive YSOs FIR 1-6 in NGC 2024. These objects are seen prominently in the dust submillimeter continuum emission (e.g. Mezger et al 1992), but the low-lying lines of $\mathrm{C}^{18} \mathrm{O}, \mathrm{CS}$, and $\mathrm{NH}_{3}$ do not show any enhancements at the positions of the YSOs. Interferometer observations by Wilson et al (1995) and Wiesemeyer et al (1997) confirm the offset between the continuum and line emission, suggesting depletions of an order of magnitude. However, Chandler \& Carlstrom (1996) pointed out that the dust and gas observations can be reconciled if the dust temperatures are substantially higher than assumed by Mezger et al. Recent determinations of the gas temperatures appear to support this hypothesis (Mangum et al 1997). The analysis is further complicated by the possibility that the dust emissivity may change within the condensations. Interferometric observations of high-frequency lines and continuum are needed to settle this issue. The chemistry in NGC 2024 has been modeled by Charnley (1998), who suggests additional chemical tests of this scenario.

The ice composition in the envelopes has also been studied in much more detail for high-mass YSOs than for their low-mass counterparts, starting with the infrared observations of Willner et al (1982). With the advent of ISO, it is now possible to obtain a nearly complete inventory of these ices. Table 1 includes a summary of the derived abundances, using the deeply embedded sources NGC 7538 IRS9, W 33A, and RAFGL 7009S as templates (d'Hendecourt et al 1996, Whittet et al 1996) (see also Figure 2). Even with the caveat that infrared data only probe abundances down to $\sim 0.5 \%$ of $\mathrm{H}_{2} \mathrm{O}$ ice $\left(\sim 10^{-7}\right.$ of $\mathrm{H}_{2}$ ), the mantle composition is remarkably simple, consisting mostly of species that result from hydrogenation and oxidation of $\mathrm{O}, \mathrm{C}, \mathrm{N}$, and $\mathrm{CO}$. The principle mystery concerns the identification of the XCN 4.62- $\mu$ m band (Lacy et al 1984, 
Table 1 Observed abundances $X / \mathrm{H}_{2}$ in Orion and comparison with ices and comets

\begin{tabular}{|c|c|c|c|c|c|c|}
\hline Species & $\begin{array}{l}\text { Extended } \\
\text { Ridge }\end{array}$ & Plateau & Hot Core ${ }^{a}$ & Ices & Comets & References $^{\mathrm{b}}$ \\
\hline $\mathrm{CO}$ & $9(-5)^{\mathrm{c}}$ & $1(-4)$ & $1(-4)$ & $(0.5-10)(-6)$ & $(1-10)(-6)$ & $1,1,1,2,3$ \\
\hline $\mathrm{H}_{2} \mathrm{O}$ & $<1(-7)$ & $>1(-5)$ & $>1(-5)$ & $5(-5)^{d}$ & $5(-5)^{d}$ & $4,5,6,2,3$ \\
\hline $\mathrm{H}_{2} \mathrm{CO}$ & $2(-9)$ & $1(-7)$ & $1(-8)$ & $\lesssim 2(-6)$ & $(0.1-2)(-6)$ & $7,1,7,2,3$ \\
\hline $\mathrm{CH}_{3} \mathrm{OH}$ & $8(-9)$ & $\lesssim 1(-7)$ & $2(-7)$ & $4(-6)$ & $(0.5-3)(-6)$ & $7,7,1,2,3$ \\
\hline $\mathrm{HCOOH}$ & $<2(-9)$ & $<1(-9)$ & $8(-10)$ & $\lesssim 1(-6)$ & $1(-7)$ & $1,11,1,2,3$ \\
\hline $\mathrm{HCOOCH}_{3}$ & $<2(-9)$ & $<3(-9)$ & $1(-8)$ & - & $2(-8)$ & $1,1,1,-, 3$ \\
\hline $\mathrm{NH}_{3}$ & $1(-8)$ & $1(-7)$ & $8(-7)$ & $<2(-6)$ & $(2-6)(-7)$ & $8,15,8,2,3$ \\
\hline $\mathrm{HCN}$ & $3(-8)$ & $9(-7)$ & $4(-7)$ & $<3(-6)$ & $1(-7)$ & $7,7,7,9,3$ \\
\hline $\mathrm{XCN} / \mathrm{HNCO}^{\mathrm{e}}$ & $2(-9)$ & $<1(-10)$ & $6(-9)$ & $\sim 1(-6)$ & $1(-7)$ & $10,11,10,2,3$ \\
\hline $\mathrm{CH}_{3} \mathrm{CN}$ & $5(-10)$ & $9(-9)$ & $2(-8)$ & - & $1(-8)$ & $7,7,7,-, 17$ \\
\hline $\mathrm{C}_{2} \mathrm{H}_{5} \mathrm{CN}$ & $<8(-10)$ & $4(-8)$ & $3(-8)$ & - & $<5(-9)$ & $1,1,7,-, 3$ \\
\hline $\mathrm{CS}$ & $1(-8)$ & $4(-9)$ & $1(-8)$ & - & $5(-8)$ & $1,1,12,-, 17$ \\
\hline SO & $1(-9)$ & $2(-7)$ & $5(-8)$ & - & - & $7,7,7,-,-$ \\
\hline $\mathrm{SO}_{2}$ & $2(-10)$ & $3(-7)$ & $6(-8)$ & $<1(-7)$ & $1(-7)$ & $7,7,7,2,3$ \\
\hline OCS & $3(-10)$ & $8(-8)$ & $5(-8)$ & $2(-8)$ & $5(-8)$ & $7,7,7,2,3$ \\
\hline $\mathrm{H}_{2} \mathrm{~S}$ & $<1(-9)$ & $1(-7)$ & $\lesssim 1(-7)$ & $<1(-7)$ & $1(-7)$ & $-, 10,11,2,3$ \\
\hline $\mathrm{SiO}$ & $<5(-10)$ & $3(-8)$ & $6(-8)$ & - & - & $1,7,7,-,-$ \\
\hline $\mathrm{CO}_{2}$ & - & $(2-10)(-7)$ & - & $8(-6)$ & $(1-10)(-6)$ & $-, 13,-, 2,3$ \\
\hline $\mathrm{CH}_{4}$ & - & $(0.5-1)(-6)^{\mathrm{f}}$ & - & $(0.5-1)(-6)$ & $(1-5)(-7)$ & $-, 14,-, 2,3$ \\
\hline $\mathrm{C}_{2} \mathrm{H}_{2}$ & - & $(3-10)(-7)$ & - & $<5(-6)$ & $(2-5)(-7)$ & $-, 15,-, 16,3$ \\
\hline $\mathrm{C}_{2} \mathrm{H}_{6}$ & - & - & - & $<2(-7)$ & $(2-5)(-7)$ & $-,-,-, 16,3$ \\
\hline
\end{tabular}

aThe values for N-containing species refer to the "hot core"; those for O-bearing molecules to the compact ridge (see text).

${ }^{b}$ References refer to each of the five columns individually.

${ }^{\mathrm{c}}$ Notation $\mathrm{a}(-\mathrm{b})$ indicates $a \times 10^{-b}$.

${ }^{\mathrm{d} A b u n d a n c e}$ fixed at $5(-5)$. The abundances for ices are typical for deeply embedded sources such as NGC 7538 IRS9 and W 33A. The abundances for comets refer mostly to Hyakutake and Hale-Bopp.

${ }^{e}$ Values for Orion and comets refer to HNCO. If the XCN ice band is due to $\mathrm{OCN}^{-}$(Schutte \& Greenberg 1997), it is expected to evaporate as HNCO.

${ }^{\mathrm{f}}$ Typical value for other sources, no limits for Orion.

References: 1. Sutton et al 1995; 2. Tielens \& Whittet 1997, Ehrenfreund et al 1997b, Schutte 1998; 3. BockeléeMorvan 1997, Crovisier 1998; 4. Tauber et al 1996; 5. Cernicharo et al 1994; 6. Gensheimer et al 1996; 7. Wright et al 1996; 8. Hermsen et al 1988, Jacq et al 1990; 9. WA Schutte, private communication; 10. Blake et al 1987; 11. Blake et al 1996; 12. Chandler \& Wood 1997; 13. AMS Boonman, private communication, infrared absorption; 14. Boogert et al 1998, infrared absorption; 15. Evans et al 1991, infrared absorption; 16. Boudin et al 1998; 17. Biver et al 1997.

Bernstein et al 1995, Schutte \& Greenberg 1997) and the strong 6.8- $\mu \mathrm{m}$ band; although $\mathrm{CH}_{3} \mathrm{OH}$ contributes to a fraction of the $6.8-\mu \mathrm{m}$ absorption, the main carrier remains unidentified (Schutte et al 1996). As the quality of the ISO data reduction improves, identification or stringent upper limits of other minor species can be obtained (e.g. Boudin et al 1998, Schutte et al 1998). Major species that are expected to be present in ices but that cannot (yet) be observed are $\mathrm{O}_{2}, \mathrm{~N}_{2}$, and $\mathrm{NH}_{3}$ (see Section 4). 
The ices toward high-mass objects have a smaller fraction of nonpolar $\mathrm{CO}$ than the low-mass cases. The $\mathrm{H}_{2} \mathrm{O} 3-\mu \mathrm{m}$ ice profiles also show evidence for heating up to $70 \mathrm{~K}$ (Smith et al 1989). Some of the high-mass objects like AFGL 2591 have warmed such a large fraction of their envelope that all solid $\mathrm{CO}$ and part of the $\mathrm{H}_{2} \mathrm{O}$ have evaporated, although some $\mathrm{H}_{2} \mathrm{O}$ and $\mathrm{CO}_{2}$ ice is still present (van Dishoeck 1998c). XCN is not detected toward these sources, suggesting that it is a transient molecule in the icy mantles. Solid $\mathrm{CO}_{2}$ is seen toward all objects and is present in both the polar and apolar phases, with evidence for heating and segregation into a pure $\mathrm{CO}_{2}$ phase (Ehrenfreund et al 1998). At first glance, the pattern of abundances toward the dozen high-mass objects studied to date is remarkably similar, indicating that the resulting ices are not very sensitive to the details of the collapse. Closer inspection reveals subtle differences in the abundances of minor species and their line profiles. As discussed in Section 7.3, the different types of ices in the envelope reflect the temperature and density profiles in the collapsing envelopes.

A new probe of the evolutionary state of the YSOs is provided by the ratios of the solid-state and gas-phase abundances derived from ground-based and ISO infrared spectroscopy. Mitchell et al (1990) performed high-resolution gas-phase $\mathrm{CO}$ and ${ }^{13} \mathrm{CO}$ absorption line observations, showing the presence of both cold $\left(T_{\text {kin }}<60 \mathrm{~K}\right)$ and hot $\left(T_{\text {kin }}=120-1000 \mathrm{~K}\right)$ gas along the same lines of sight for which ices have been observed. The hot, dense gas appears to contain high abundances of $\mathrm{C}_{2} \mathrm{H}_{2}, \mathrm{HCN}$, and $\mathrm{CH}_{4}$ (Lacy et al 1989, 1991, Evans et al 1991, Carr et al 1995, Boogert et al 1998; F Lahuis \& EF van Dishoeck, in preparation), as well as $\mathrm{H}_{2} \mathrm{O}$ (Helmich et al 1996a, van Dishoeck \& Helmich 1996). Gas-phase $\mathrm{CO}_{2}$, however, has a surprisingly low abundance (van Dishoeck et al 1996). Clear variations in the gas/solid ratios of two orders of magnitude are seen for various objects (van Dishoeck et al 1996, 1998, Dartois et al 1998; see Figure 5), indicating the development of a hot core in the inner envelope in which the ices are evaporated and where the high temperatures drive the gas-phase oxygen into $\mathrm{H}_{2} \mathrm{O}$.

\section{THE INFLUENCE OF OUTFLOWS ON CHEMISTRY}

\subsection{Models}

One of the earliest, and initially puzzling, observational signposts of star formation in molecular clouds was the widespread detection of outflowing supersonic gas (Snell et al 1980). That the ejection of material at high speeds from deep in the gravitational well of young stars is a necessary complement to mass accretion is now thought to arise naturally from the need to shed the vast majority of the original angular momentum content of collapsing cloud cores. The 


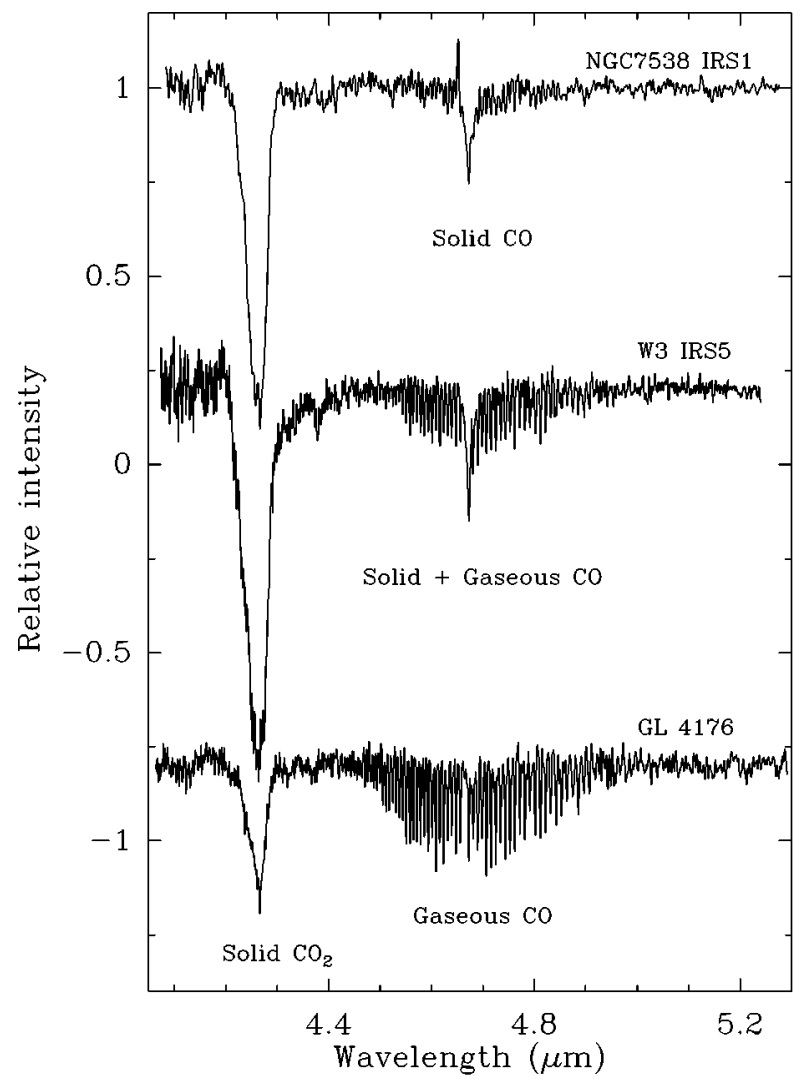

Figure 5 ISO-SWS spectra of the solid-state $\mathrm{CO}_{2}$ and solid- and gas-phase $\mathrm{CO}$ infrared absorption features toward embedded massive YSOs, illustrating the evaporation of ices and development of a "hot core" region near W 3 IRS5 and AFGL 4176. The relative ratios of these features can be used as an indicator of their evolutionary state (van Dishoeck et al 1996, 1998).

means by which the YSO/accretion disk system produces and directs the outflows is still much debated, in particular whether collimated jets or star/disk winds are responsible (Pelletier \& Pudritz 1992, Shu et al 1995). In any case, as the high-velocity (several hundred kilometers per second) material flows outward and strikes the envelope or ambient medium, shocks are driven into the originally quiescent material, thereby allowing new chemical processes to occur. Understanding the detailed nature of outflows currently forms an area of intense research, and reviews summarizing recent progress in this area may be 
found in Bachiller (1996) and in the conference proceedings edited by Beckwith et al (1996) and Reipurth \& Bertout (1997).

The chemical manifestations of these shocks can be substantial, and they depend on whether the shocks are of the $J$ (or jump) or $C$ (or continuous) type (Draine \& McKee 1993, Hollenbach 1997), which in turn depends on the shock velocity and the magnetic field strength and fractional ionization in the preshocked gas. For typical ionization fractions in dense clouds $x(\mathrm{e}) \lesssim 10^{-6}$, $C$ shocks occur at $\mathrm{v}_{s} \lesssim 40-50 \mathrm{~km} \mathrm{~s}^{-1}$. Their peak temperatures are typically 2000-3000 K, too low for molecular dissociation to occur. However, reactions with energy barriers, such as $\mathrm{O}+\mathrm{H}_{2} \rightarrow \mathrm{OH} \rightarrow \mathrm{H}_{2} \mathrm{O}$ and $\mathrm{S}+\mathrm{H}_{2} \rightarrow \mathrm{SH} \rightarrow \mathrm{H}_{2} \mathrm{~S}$, proceed rapidly in the warm dense gas, driving all available oxygen and sulfur into $\mathrm{H}_{2} \mathrm{O}$ and $\mathrm{H}_{2} \mathrm{~S}$ (e.g. Draine et al 1983). Back reactions with atomic hydrogen can destroy these species again, so that the balance between $\mathrm{O}, \mathrm{OH}$, and $\mathrm{H}_{2} \mathrm{O}$ depends on the $\mathrm{H} / \mathrm{H}_{2}$ ratio in the shock. $\mathrm{OH}$ is an important intermediary in the creation of many other species; e.g. reactions with $\mathrm{S}, \mathrm{Si}$, and $\mathrm{N}$ produce $\mathrm{SO}$, $\mathrm{SiO}$, and NO.

In $J$ shocks, the temperature is increased to such high values $\left(\sim 10^{5} \mathrm{~K}\right)$ that all molecules are dissociated (Hollenbach \& McKee 1989, Neufeld \& Dalgarno 1989a,b). In addition, ultraviolet radiation is produced, which may photodissociate molecules both in and ahead of the shocks. The molecules then reform slowly in a lengthy warm zone in the wake of the shock. Once substantial $\mathrm{H}_{2}$ has been reformed, the chemistry resembles that of the $C$ shocks. In most YSOs, a mixture of $J$ - and $C$-type shocks likely occurs.

In addition to these gas-phase processes, grain cores and their mantles are affected by the shocks. Outright destruction or thermal sputtering of grain cores by high-velocity $J$-type shocks introduces locally high abundances of refractory elements such as $\mathrm{Si}$ and $\mathrm{Fe}$, while the nonthermal sputtering of cores and ice mantles in lower velocity $C$ shocks, mostly by particles heavier than $\mathrm{He}$, can also inject a variety of both refractory and volatile species into the gas phase (Flower \& Pineau des Forêts 1994, 1995, Jones et al 1994, Flower et al 1995, 1996, Tielens 1998). Extensive one-dimensional chemical networks have been constructed for C-, N-, O-, S-, and Si-bearing species that account for the shock physics and, in the more recent models, for the interactions of gas-phase molecules with dust grains (e.g. Mitchell 1984a,b, Leen \& Graff 1988, Pineau des Forêts et al 1993, Kaufman \& Neufeld 1996, Caselli et al 1997, Schilke et al 1997b). The output from such models consistently reveals zones within the shock $\sim 100-10,000$ AU in size, with very different chemical and physical properties from the preshocked gas, with enhancements of species such as $\mathrm{OH}, \mathrm{H}_{2} \mathrm{O}, \mathrm{SiO}$, and $\mathrm{SiO}_{2}$. $\mathrm{SiO}$ is particularly increased because of the large depletion of silicon in the preshocked gas (see Section 4). Even a return of only $\sim 1 \%$ of the interstellar silicon abundance to the gas is 
sufficient to result in enhancements of orders of magnitude over the preshocked gas.

\subsection{Observations}

Mapping of nearby clouds like Orion and NGC 1333 in tracers of dense shocked gas, such as the vibrational emission from $\mathrm{H}_{2}$ (Yu et al 1997) or the high-velocity line wings of CO and CS (Sandell et al 1994, Warin et al 1996b), have shown that the cloud structure appears to be completely dominated by the outflows, which snake through the entire region and thereby sweep up material and shockcompress the gas. Bergin et al (1998) have considered the chemical "legacy," especially of $\mathrm{H}_{2} \mathrm{O}$, in the wake of such stochastic shock activity using a timedependent chemical and dynamical model.

Exacting tests of the detailed shock models in a number of deeply embedded low-mass YSOs are provided by high angular resolution observations in specific shock tracers and cooling lines (e.g. Guilloteau et al 1992, Chernin et al 1994, Blake et al 1995, Dent et al 1995, Bachiller \& Perez-Gutierrez 1997). One of the more spectacular recent examples is that of NGC 1333 IRAS 2 presented in Figure 6. First discovered by Sandell et al (1994) at the tip of a collimated CO outflow, this source appears to provide a molecular counterpart to the optical jets

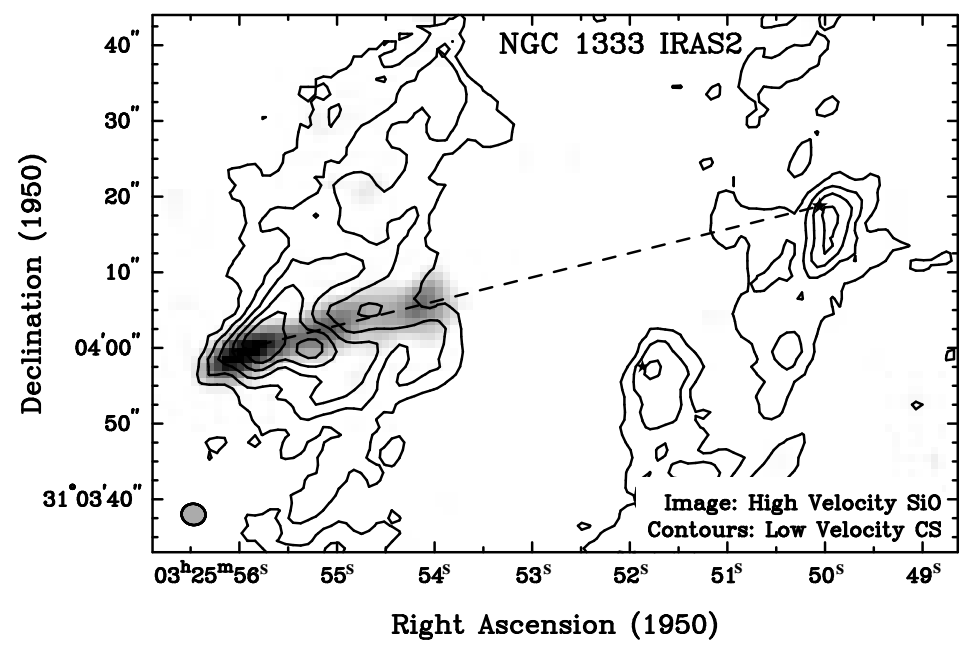

Figure 6 Image of the CS J=2-1 emission from the ambient gas (contours) and $\mathrm{SiO} \mathrm{J}=2-1$ emission from high-velocity gas ( gray scale) associated with the outflow from NGC 1333 IRAS 2. The "stars" mark the positions of the IRAS $2 \mathrm{~A}$ and $2 \mathrm{~B} \lambda=2.7-\mathrm{mm}$ continuum sources. The $\mathrm{SiO}$ emission traces the extremely collimated outflow, while the CS emission traces the outflow interaction region within the cloud as well as the collapsing cores associated with IRAS 2A and 2B (Blake 1997). 
so frequently observed in pre-main sequence objects. Combined interferometric and single-dish mapping has demonstrated that the outflow impacts dense, cold preshocked gas $\left[n\left(\mathrm{H}_{2}\right)=10^{5} \mathrm{~cm}^{-3}, T=20 \mathrm{~K}\right]$ that is subsequently heated and compressed $\left[n\left(\mathrm{H}_{2}\right)=10^{7} \mathrm{~cm}^{-3}, T=100 \mathrm{~K}\right]$ (Blake et al 1995, Warin et al 1996b), resulting in strong molecular emission from the postshocked gas, especially in $\mathrm{SiO}$. The jet-like structure traced by the high-velocity $\mathrm{SiO}$ projects back toward IRAS $2 \mathrm{~A}$ some 17,400 AU distant. While the jet itself is optically invisible, its diameter is constrained to be $\leq 500 \mathrm{AU}$ by the $\mathrm{SiO}$ and CS images, which also highlight the importance of the ambient medium in shaping the flow. For example, the low-velocity CS is aligned primarily along a north-south ridge that is distorted into a bow-shock shape by the outflow, which is remarkably similar to numerical simulations of jets driving into increasing density gradients (de Gouveia Dal Pino et al 1996, Cerqueira et al 1997).

In addition to NGC 1333 IRAS 2, other well-studied low-mass sources include NGC 1333 IRAS 4 (Blake et al 1995), L1157 (e.g. Mikami et al 1992, Avery \& Chiao 1996, Bachiller \& Perez-Gutierrez 1997), and Sandqvist 136 (Garay et al 1996). These sources have the advantage of a large spatial separation between the quiescent and shocked regions so that their chemistries can be studied separately. Care should be taken to first constrain the density in the shocked gas, since strong, broad profiles do not necessarily imply shockenhanced abundances. Nevertheless, in the $\mathrm{L} 1157$ outflow, the abundances of many species, both refractory and volatile ( $\mathrm{SiO}, \mathrm{SO}, \mathrm{CS}, \mathrm{HCN}, \mathrm{H}_{2} \mathrm{CO}, \mathrm{CH}_{3} \mathrm{OH}$ ), appear to be considerably increased by factors of 5-500 for the volatiles and up to $10^{6}$ for the most refractory components such as $\mathrm{SiO}$. Similar $\mathrm{SiO}$ enhancements have been seen in many other objects, where $\mathrm{SiO}$ also shows kinematic and spatial offsets from other species (e.g. Martín-Pintado et al 1992, McMullin et al 1994a,b, Bachiller 1996). Since the production of $\mathrm{SiO}$ requires grain core or mantle destruction, it points to the location of directly shocked material at high velocities, whereas the more volatile species $\left(\mathrm{HCN}, \mathrm{CH}_{3} \mathrm{OH}\right)$ can only survive sputtering or desorption of grain mantles at low-shock velocities of $\sim 10 \mathrm{~km} \mathrm{~s}^{-1}$ or less. Thus, much like the hot cores surrounding massive YSOs, the chemical composition of outflow/envelope interaction zones may be used to estimate the products of grain mantle chemistry. The chemical processes in the shock-heated gas differ from those in hot cores, however, because of the very high abundance of the reactive OH radical (Schilke et al 1997b) in the former, meaning that distinct chemical models must be constructed for these environments. The best-studied shock in high-mass regions is the Orion-KL plateau region, which also shows enormous enhancements of $\mathrm{SiO}, \mathrm{SO}$, and $\mathrm{SO}_{2}$ (see Section 10.1).

One of the prime predictions of all shock models in dense clouds is the copious production of $\mathrm{H}_{2} \mathrm{O}$, which contains all oxygen not locked up in $\mathrm{CO}$. 
Ground-based and airborne measurements of $\mathrm{H}_{2} \mathrm{O}$ are severely hampered by the atmosphere, but a series of observations (primarily of the Orion shock) do reveal high $\mathrm{H}_{2} \mathrm{O}$ abundances of at least $10^{-5}$ (e.g. Cernicharo et al 1994, Zmuidzinas et al 1995, Timmermann et al 1996). Far-infrared observations of the dominant shock coolants, including $\mathrm{O}, \mathrm{OH}$, and $\mathrm{H}_{2} \mathrm{O}$, also are possible with ISO, although at low spatial and spectral resolution (Nisini et al 1996, Ceccarelli et al 1998). For the shock in the low-mass Herbig-Haro object HH54, Liseau et al (1996) found an $\mathrm{H}_{2} \mathrm{O}$ abundance of $\sim 10^{-5}$, which contributes some $20 \%$ of the total cooling.

Masers have also been widely observed to be associated with star-forming regions (see Menten 1997, Hollenbach 1997 for reviews). $\mathrm{H}_{2} \mathrm{O}$ masers most likely originate in outflows because they require densities of $10^{7}-10^{9} \mathrm{~cm}^{-3}$ and temperatures of $\sim 500 \mathrm{~K}$ to be excited. In contrast, the $\mathrm{OH}$ and class II $\mathrm{CH}_{3} \mathrm{OH}$ masers can be generated in lower density $\left(\sim 10^{7} \mathrm{~cm}^{-3}\right)$ hot cores near ultracompact $\mathrm{H}$ II regions, in which their abundances are enhanced owing to evaporation of $\mathrm{CH}_{3} \mathrm{OH}$ and $\mathrm{H}_{2} \mathrm{O}$ from icy mantles (see Section 9).

\section{CHEMISTRY IN HOT CORES}

\subsection{Observations}

When a young star begins to heat the inner envelope that surrounds it by radiation and/or shocks to temperatures above $\sim 100 \mathrm{~K}$, a very distinctive chemically rich phase ensues. These so-called hot cores have been mostly observed for massive stars and are small $(<0.1 \mathrm{pc})$, dense $\left[n\left(\mathrm{H}_{2}\right)>10^{6} \mathrm{~cm}^{-3}\right]$, and warm $\left(T_{k}>100 \mathrm{~K}\right)$ regions of a few hundred $M_{\odot}$ (Walmsley 1992). Most of them have associated masers (e.g. Hofner \& Churchwell 1996, Codella et al 1997) and show signs of outflow activity (e.g. Shepherd \& Churchwell 1996, Acord et al 1997, Cesaroni et al 1997, Hunter et al 1997). They are usually near (ultracompact) H II regions (Wood \& Churchwell 1989) but are not necessarily associated with them. In fact, the free-free continuum emission from hot cores is often undetectable, suggesting that these objects are at an early stage before the $\mathrm{H}$ II region starts to develop. A well-known example is the $\mathrm{W} 3\left(\mathrm{H}_{2} \mathrm{O}\right)$ hot core (Turner \& Welch 1984), located $\sim 7^{\prime \prime}(0.08 \mathrm{pc})$ from the more evolved W $3(\mathrm{OH}) \mathrm{H}$ II region.

Chemically, hot cores are characterized by high abundances of fully hydrogenated molecules such as $\mathrm{H}_{2} \mathrm{O}$ (e.g. Cernicharo et al 1990, Gensheimer et al 1996), $\mathrm{NH}_{3}$ (e.g. Genzel et al 1982, Pauls et al 1983, Cesaroni et al 1992, 1994, Wyrowski \& Walmsley 1996) and $\mathrm{H}_{2} \mathrm{~S}$ (Minh et al 1990, 1991), along with a rich variety of complex organic molecules, ranging from methanol and ethanol (e.g. Menten et al 1988, Millar et al 1995) to $\mathrm{CH}_{3} \mathrm{CN}$ (e.g. Olmi et al 1996), dimethyl ether, methyl formate, and ethyl cyanide (e.g. Miao \& Snyder 1997, Ohishi 1997, Hatchell et al 1998b). In addition, high deuterium 
fractionation is found in species such as HDO (Jacq et al 1990, Gensheimer et al 1996, Helmich et al 1996b), DCN (Mangum et al 1991), HDCO (Loren \& Wootten 1985), $\mathrm{D}_{2} \mathrm{CO}$ (Turner 1990), and $\mathrm{CH}_{2} \mathrm{DCN}$ (Gerin et al 1992). Recent reviews have been given by Walmsley \& Schilke (1993) and Ohishi (1997).

The sample of sources studied to date consists of the archetypical Orion (see Section 10.1, Table 1) and SgrB2(N) (Kuan \& Snyder 1994, 1996, Miao et al 1995, Mehringer et al 1997) hot cores; sources such as W 3( $\left.\mathrm{H}_{2} \mathrm{O}\right)$, W 49, W 51; and objects near ultracompact H II regions, such as G34.3+0.15 (Macdonald et al 1996, Mehringer \& Snyder 1996), G10.47, and G9.62 (Hofner et al 1996). In some cases, the ultracompact H II regions are located in more extended, warm molecular material without a denser, compact condensation (Hatchell et al 1998b). Either these regions have hot cores that are too small $\left(<1^{\prime \prime}\right)$ to be picked up by the single-dish surveys, or they have been dispersed already, indicating a later phase in their evolution. Indeed, chemistry may be particularly useful in distinguishing their evolutionary stage, as discussed in Section 9.3. Hot cores can also be studied through infrared absorption line observations (e.g. Mitchell et al 1990, van Dishoeck et al 1998; see Section 7.4).

Hot cores show strong, rich spectra and are therefore prime targets for spectral line surveys. Unbiased single-dish spectral scans have been performed for only a handful of objects, including Orion (e.g. Johansson et al 1984, Sutton et al 1985, 1995, Blake et al 1986, 1987, Jewell et al 1989, Ziurys \& McGonagle 1993, Schilke et al 1997a), SgrB2 (e.g. Cummins et al 1986, Sutton et al 1991, Turner 1991, Nummelin 1996), W 3( $\left.\mathrm{H}_{2} \mathrm{O}\right)$ (Helmich \& van Dishoeck 1997), and G34.3+0.15 (Macdonald et al 1996). Such surveys provide a wealth of information on the chemistry, but accurate determination of abundances is complicated by the ill-determined source structure. While there is no doubt that the abundances of the complex organics are enhanced by several orders of magnitude in the compact hot cores, their actual values are still uncertain by up to an order of magnitude.

\subsection{Models}

The observed abundances of the hot core molecules listed in Table 1 are much larger than can be produced by low-temperature ion-molecule gas-phase chemistry. The only plausible models developed to date are those in which the chemistry is driven by the evaporation of icy grain mantles. Blake et al (1987) suggested that the large abundances of oxygen-bearing organics in the Orion compact ridge could be due to injection of $\mathrm{H}_{2} \mathrm{O}$ into the ridge gas, leading to the formation of protonated methanol through reaction with $\mathrm{CH}_{3}^{+}$, which then reacts with a variety of neutral species to produce the observed organics. Subsequent models by Millar et al (1991a) showed that $\mathrm{CH}_{3} \mathrm{OH}$, rather than 
$\mathrm{H}_{2} \mathrm{O}$, injection is needed to drive a rapid gas-phase chemistry and approach the measured abundances. Recent reviews have been given by Millar (1997) and Tielens \& Charnley (1997).

The first comprehensive models for hot cores were developed by Brown et al (1988), who followed the gas-phase chemistry during the collapse from a diffuse medium up to the density when virtually all species have condensed onto the grains. They assumed that simple hydrogenation reactions on the surfaces result in large abundances of hydrides in the ices and that some heating event associated with the new star subsequently liberates the icy mantles into the gas. The model by Brown et al had little chemical evolution in the hot gas because of their adopted high $\mathrm{Mg}$ abundance (and consequent high electron abundance), which resulted in low ion abundances and thus a slow ion-molecule chemistry. Owing to the uncertainties in grain-surface chemistry, subsequent hot core models took a more pragmatic approach by using the observed ice mantle composition from infrared spectroscopy as the starting point. Charnley et al (1992) showed that the observed abundances in the Orion hot cores could be reproduced if a mixture of simple ices containing $\mathrm{H}_{2} \mathrm{O}, \mathrm{CO}, \mathrm{CH}_{3} \mathrm{OH}, \mathrm{NH}_{3}$, and/or $\mathrm{HCN}$ is evaporated into the hot gas (see Figure 7).

There are two classes of reactions that break down the evaporated molecules and initiate the hot core chemistry: (a) reactions with atomic hydrogen, e.g. $\mathrm{H}_{2} \mathrm{~S}+\mathrm{H} \rightarrow \mathrm{SH}+\mathrm{H}_{2} ;(b)$ reactions with protonated molecules followed by dissociative recombination, e.g. $\mathrm{H}_{2} \mathrm{O}+\mathrm{H}_{3}^{+} \rightarrow \mathrm{H}_{3} \mathrm{O}^{+}$and $\mathrm{H}_{3} \mathrm{O}^{+}+e \rightarrow \mathrm{OH}$ or $\mathrm{O}$. The key step in the formation of the oxygen-containing organics is the protonation of the alcohols, followed by transfer of an alkyl $\left(-\mathrm{CH}_{3}\right)$ group to a neutral molecule, a process that is well-studied in the laboratory (e.g. Karpas \& Meotner 1989). For example, $\mathrm{CH}_{3} \mathrm{OH}+\mathrm{H}_{3}^{+}, \mathrm{H}_{3} \mathrm{O}^{+}$, or $\mathrm{HCO}^{+} \rightarrow \mathrm{CH}_{3} \mathrm{OH}_{2}^{+}$; $\mathrm{CH}_{3} \mathrm{OH}_{2}^{+}+\mathrm{CH}_{3} \mathrm{OH} \rightarrow\left(\mathrm{CH}_{3}\right)_{2} \mathrm{OH}^{+}$; and $\left(\mathrm{CH}_{3}\right)_{2} \mathrm{OH}^{+}+e \rightarrow \mathrm{CH}_{3} \mathrm{OCH}_{3}$. The detailed chemistry of interstellar alcohols leading to large ethers and esters has been discussed by Charnley et al (1995).

The hot core chemistry has been extended to include additional N- (Kress \& Charnley 1996), S- (Charnley 1997), Si- (MacKay 1995), and P-bearing (Charnley \& Millar 1994) species. In these models, it is assumed that most of the $\mathrm{S}, \mathrm{Si}$, and $\mathrm{P}$ leaves grain surfaces in the form of $\mathrm{H}_{2} \mathrm{~S}, \mathrm{SiH}_{4}$, and $\mathrm{PH}_{3}$, which are rapidly destroyed by reactions with atomic hydrogen to yield $\mathrm{S}, \mathrm{Si}$, and $\mathrm{P}$ at temperatures of a few hundred Kelvin because the reactions have low energy barriers. The resulting atoms can then react with species such as $\mathrm{OH}$ and $\mathrm{O}_{2}$ in the warm gas to form $\mathrm{SO}, \mathrm{SO}_{2}, \mathrm{SiO}$, and $\mathrm{PO}$. The outcome of these chemistries is linked to the oxygen chemistry, in particular the relative abundances of $\mathrm{O}, \mathrm{OH}$, and $\mathrm{H}_{2} \mathrm{O}$, which depend sensitively on temperature. At $T \geq 230 \mathrm{~K}$, most of the oxygen is driven into $\mathrm{H}_{2} \mathrm{O}$ and virtually no $\mathrm{O}$ and $\mathrm{O}_{2}$ are available, preventing the 

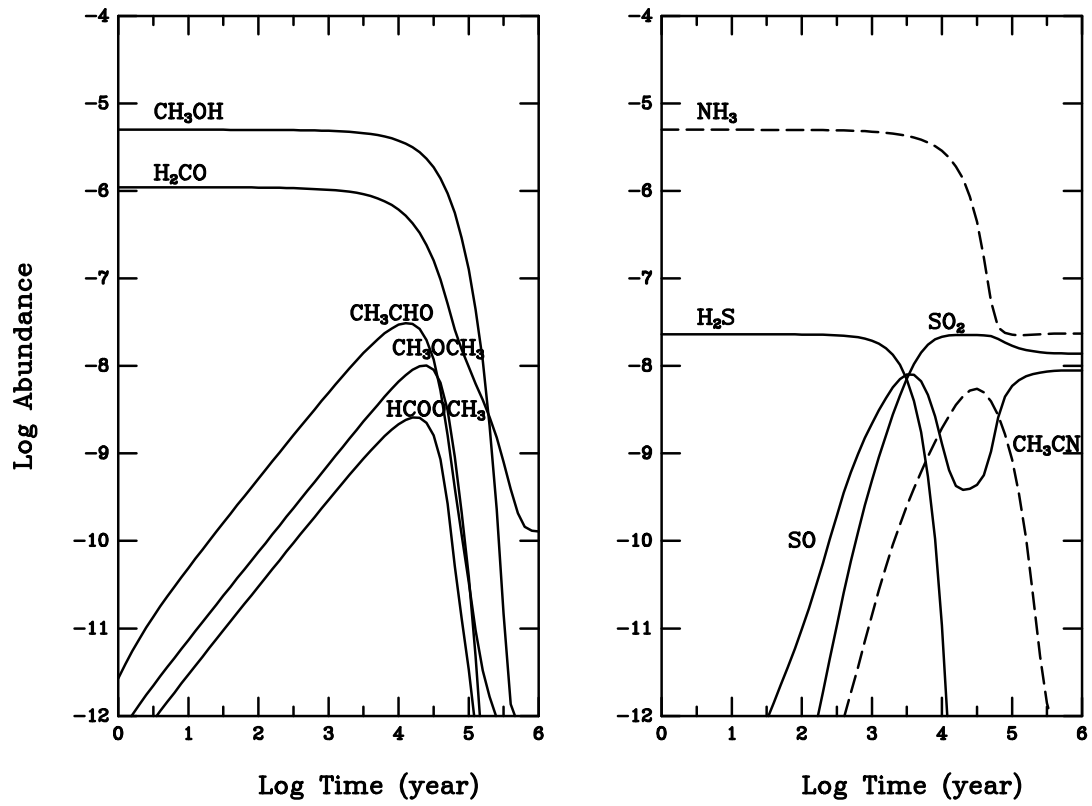

Figure 7 Models of the gas-phase chemical evolution during the hot core phase for $T=200 \mathrm{~K}$. At $t=0$, molecules such as $\mathrm{CH}_{3} \mathrm{OH}, \mathrm{NH}_{3}$, and $\mathrm{H}_{2} \mathrm{~S}$ are evaporated from the grain mantles into the gas phase, where they drive a rapid chemistry leading to more complex organic molecules such as $\mathrm{CH}_{3} \mathrm{OCH}_{3}, \mathrm{HCOOCH}_{3}, \mathrm{CH}_{3} \mathrm{CN}$, and $\mathrm{SO}_{2}$. The abundances peak after $10^{4}-10^{5}$ years (based on Charnley et al 1992, Charnley 1997).

formation of, for example, $\mathrm{SO}_{2}$ (Charnley 1997). $\mathrm{O}$ and $\mathrm{O}_{2}$ also destroy reactive species such as $\mathrm{CN}$, which is an important building block in the formation of $\mathrm{HC}_{3} \mathrm{~N}$ through its reaction with evaporated $\mathrm{C}_{2} \mathrm{H}_{2}$. Larger cyanopolyynes are not effectively made in the hot cores unless the mantles contain highly unsaturated hydrocarbons such as $\mathrm{C}_{4} \mathrm{H}_{2}$.

Based on the models, three kinds of species can be distinguished in hot cores: (a) molecules that are produced in the precollapse, cold dense cloud chemistry by ion-molecule reactions and then "frozen" onto the grains and released into the gas-phase largely unaltered (e.g. $\left.\mathrm{C}_{2} \mathrm{H}_{2}, \mathrm{CO}\right) ;(b)$ molecules that are made by grain-surface reactions and then released as "first generation" species into the gas (e.g. $\mathrm{H}_{2} \mathrm{O}, \mathrm{NH}_{3}, \mathrm{H}_{2} \mathrm{~S}, \mathrm{CH}_{3} \mathrm{OH}$, etc); and (c) molecules that are produced by the rapid gas-phase chemistry in the hot core between evaporated molecules, which leads to "second generation" species (e.g. $\mathrm{CH}_{3} \mathrm{OCH}_{3}, \mathrm{HC}_{3} \mathrm{~N}$, etc). An active point of discussion is the determination of which molecules are first and second generation. For example, $\mathrm{C}_{2} \mathrm{H}_{5} \mathrm{OH}$ is most likely first rather than 
second generation. The observed composition of ice mantles listed in Table 1 provides direct information on the most abundant first-generation species, but only down to $\sim 0.5 \%$ of solid $\mathrm{H}_{2} \mathrm{O}$, corresponding to $\sim 10^{-7}$ with respect to $\mathrm{H}_{2}$. Hot core observations may provide much tighter constraints on the composition and complexity of the minor species in grain mantles, once the modeling of their chemistry is well understood. Additional guidance can be obtained from observations of molecules in the comae of comets Hale-Bopp and Hyakutake, assuming that the chemical composition of cometary comae largely resembles that of interstellar ices (see Section 11).

It is thought that the observed high $\mathrm{D} / \mathrm{H}$ fractionation largely reflects the $\mathrm{D} / \mathrm{H}$ ratios in the ices (e.g. Plambeck \& Wright 1987). This can result from two different processes, both of which are likely to be operative. First, gas-phase deuterium (D) fractionation is substantial in the cold collapsing cloud through low-temperature ion-molecule reactions (e.g. Millar et al 1989). Second, reactions with D on grain surfaces can lead to enhanced abundances of deuterated molecules because the gas-phase atomic $\mathrm{D} / \mathrm{H}$ ratio in a dense molecular cloud is much larger than the overall isotope ratio (Tielens 1983). Rodgers \& Millar (1996) have studied the D chemistry in the hot core phase and concluded that the $\mathrm{D} / \mathrm{H}$ ratios of the evaporated species are not much changed in the hot gas for a period of $\sim 10^{4}$ years.

The above models all calculate the hot core chemistry for a single temperature and density. In reality, the hot core is embedded in an extended envelope or halo with varying density and temperature, so that the depth-dependence of the models needs to be considered. Millar et al (1997b) have presented a comprehensive time- and depth-dependent treatment of the chemistry in G34.3+0.15 based on a three-component physical model of the region that is constrained by observations. The model clearly delineates the contributions of each of the components to the total column density of a given molecule. For example, $\mathrm{HCO}^{+}$is almost entirely in the halo, whereas $\mathrm{CH}_{3} \mathrm{OCH}_{3}$ and $\mathrm{H}_{2} \mathrm{~S}$ are almost entirely in the (ultra)compact hot core.

\subsection{Chemical Clocks}

Figure 7 shows the time-dependence of the abundances of typical hot core molecules since their injection from grains. The oxygen-containing species peak at 5,000-20,000 years, whereas the nitrogen-bearing molecules peak somewhat later. The time scale is determined by the rate at which the first-generation molecules react, which in turn depends primarily on the temperature of the gas (for reactions with $\mathrm{H}$ ) and the ionization fraction (for proton-transfer reactions). Comparison with the observed abundances leads to an estimated age of the hot core region, which should be close to that of the YSO. Although this comparison is still semiquantitative, typical values of $\sim 6,000$ [Orion compact 
ridge, W 3( $\left.\mathrm{H}_{2} \mathrm{O}\right)$ ] (Charnley 1997; FP Helmich, TJ Millar, EF van Dishoeck, in preparation) and $\sim 3,000-10,000$ years (G34.3+0.15) (Millar et al 1997b) are found. The ages of the envelopes in which they are embedded are typically $\sim 10^{5}$ years. Hatchell et al (1998a) also showed that the observed DCN/HCN ratios in hot cores of a few times $10^{-3}$ can be reached in $\sim 10^{4}$ years. The abundance ratios of S-bearing species, especially those of $\mathrm{H}_{2} \mathrm{~S}, \mathrm{SO}$, and $\mathrm{SO}_{2}$, may be particularly valuable as a chemical "clock" (Charnley 1997), a technique that has been applied by Hatchell et al (1998b) to several regions. The inferred ages range from 5,000 to 30,000 years and agree remarkably well with the dynamical times estimated for the outflows. This agreement may be fortuitous, however, since many of the cores contain multiple YSOs, each of which may drive an (time-dependent) outflow that cannot be distinguished with singledish observations alone. Also, enhancements of sulfur-bearing species may arise in shocks. High-resolution interferometric observations are essential to disentangling these effects.

At later times, the hot cores are probably fragmented into clumps and dispersed owing to the development and expansion of the H II region (e.g. Williams et al 1996), photoevaporation (Lizano et al 1996), winds, and outflows (Churchwell 1990). Part of the hot core material may end up in the new star, although little is known about mass accretion in this phase. Once the hot core is dispersed, the ultraviolet radiation from the young massive star can affect the surrounding envelope by setting up a neutral PDR outside the $\mathrm{H}$ II region. The physics and chemistry of such PDRs have been discussed extensively in the recent reviews by Sternberg et al (1997) and Hollenbach \& Tielens $(1997,1998)$ and are not reiterated here. The gas-phase PDR chemistry is characterized by simple species and large abundances of radicals such as $\mathrm{CN}$ and $\mathrm{C}_{2} \mathrm{H}$. The ices will be further processed by the intense ultraviolet radiation, resulting in $\mathrm{C}$-rich but $\mathrm{H}$ - and $\mathrm{O}$-poor organic refractory material.

\section{EXAMPLES}

Single-dish observations of YSOs usually contain a mixture of the various components (envelope, outflow, hot core) discussed in the previous sections in the observing beam. However, even such global chemical characteristics can provide useful information on their evolutionary state, as illustrated for the case of three massive YSOs in W 3 by Helmich \& van Dishoeck (1997). In the following, a well-studied high- and low-mass YSO region is discussed.

\subsection{Orion-IRc2}

The Orion-IRc2/KL region is the best-studied area of massive star formation in the Galaxy. Its close proximity and extraordinary brightness have enabled pioneering observations that, historically, have played a pivotal role in our 
understanding of other massive YSOs. Single-dish studies centered on IRc2 have revealed a number of distinct kinematic components in the observed line profiles that could not be separated spatially. These were termed the extended ridge, compact ridge, hot core, and plateau components, where the ordering runs from the smallest to largest linewidths observed for the components (see Johansson et al 1984, Blake et al 1987, Genzel \& Stutzki 1989 for overviews). Excitation conditions in the different subregions vary greatly, and substantial chemical differences were inferred from the observed line intensities, which are summarized in Table 1. The plateau source is thought to be formed by outflow interactions similar to those outlined in Section 8, with species such as $\mathrm{SiO}$, $\mathrm{SO}, \mathrm{SO}_{2}$, and $\mathrm{H}_{2} \mathrm{~S}$ being very prominent and a great many other molecules showing enhanced emission at velocities well removed from systemic. The compact ridge and hot core sources are each hot cores, which, like the plateau source, must be viewed through the extended ridge or surrounding lower density molecular cloud, material that has been studied in detail by Ungerechts et al (1997). Early studies of the compact ridge and hot core discovered sizeable differences in their chemical composition, with the compact ridge rich in complex O-bearing organics such as $\mathrm{CH}_{3} \mathrm{OH}, \mathrm{HCOOCH}_{3}$, and $\left(\mathrm{CH}_{3}\right)_{2} \mathrm{O}$, while the hot core emission was dominated by N-bearing molecules like $\mathrm{NH}_{3}$, $\mathrm{CH}_{3} \mathrm{CN}$, and $\mathrm{C}_{2} \mathrm{H}_{5} \mathrm{CN}$ (Blake et al 1987). In subsequent studies, the differences between the two sources have become less prominent (Sutton et al 1995).

Over the past few years, it has become possible to image sources as bright as Orion IRc2 at or below $1^{\prime \prime}$ with radio and millimeter-wave interferometers (Chandler \& DePree 1995, Wright et al 1995, 1996, Blake et al 1996, Chandler \& Wood 1997). An example of these studies may be found in Figure 8, which presents 1.3-mm observations of the velocity-integrated emission from $\mathrm{SiO}$, $\mathrm{C}_{2} \mathrm{H}_{5} \mathrm{CN}, \mathrm{HC}_{3} \mathrm{~N}, \mathrm{HNCO}, \mathrm{HCOOCH}_{3}$, and $\mathrm{HDO}$, overlayed on the dust emission image. These studies have better clarified the chemical composition of the two hot cores and the plateau source by isolating them spatially and by filtering out emission from the extended ridge. Significantly, a number of new discoveries about the Orion hot core in molecules such as methanol and in additional families such as S-bearing species that could not be isolated in the single-dish work can now be compared to models of hot core chemistry. Another significant development is the use of mid-infrared observations to probe the plateau/hot core abundances of species such as $\mathrm{C}_{2} \mathrm{H}_{2}, \mathrm{HCN}, \mathrm{OCS}$, and $\mathrm{NH}_{3}$ in absorption toward IRc2 (Evans et al 1991). The resulting abundances are summarized in Table 1, and the various determinations agree well, typically within a factor of a few.

The hot core itself lies nearest to the exciting source in the region (radio source I, Menten \& Reid 1995). The complex spatial patterns observed in Figure 8 suggest variations in both the physical conditions and grain mantle composition on small scales, with the clump exteriors currently being evaporated and/or ablated 


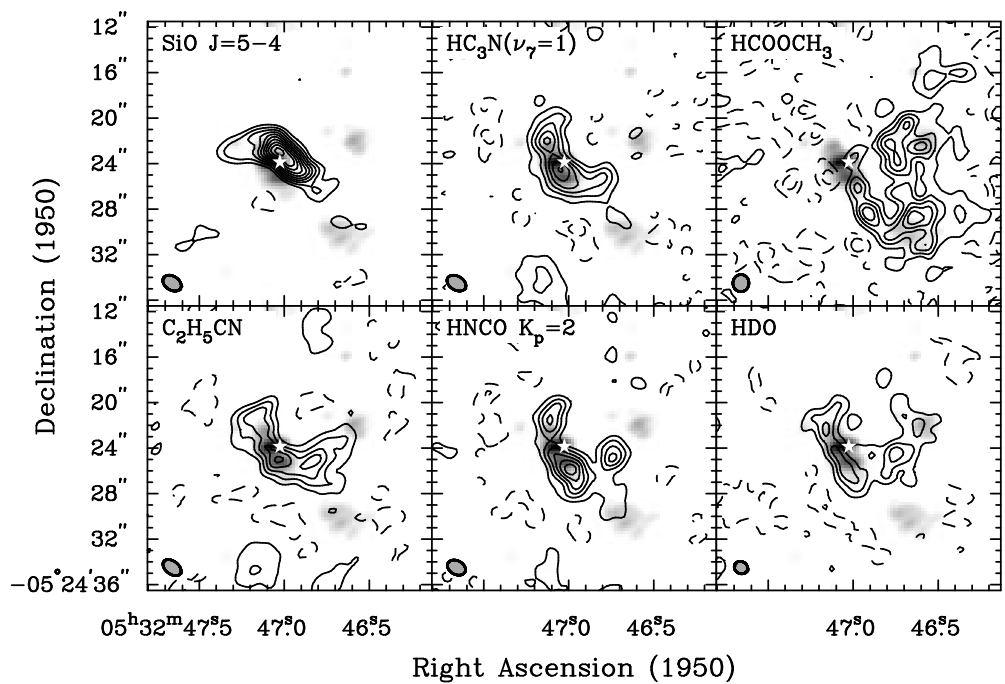

Figure 8 A multipanel image summarizing various aspects of the hot core chemistry observed toward Orion KL with the Owens Valley millimeter array at $\lambda=1.3 \mathrm{~mm}$. In each panel, the gray scale presents the continuum emission at $1.5^{\prime \prime} \times 1.0^{\prime \prime}$ resolution, whereas the contours depict the maps obtained for selected molecules. The white star marks the position of radio source I and the $\mathrm{SiO}(v=1)$ maser ring. The "compact ridge" region lies $\sim 8^{\prime \prime}$ to the southwest (Blake et al 1996).

by embedded source(s). Caselli et al (1993) have put forward a plausible model of how such differentiation could have occurred, by assuming a temperature gradient in the original collapsing cloud. In the hot core region close to IRc2, the temperature may not have been low enough for the volatile $\mathrm{CO}$ to freeze out onto the grains, thereby preventing the formation of solid $\mathrm{CH}_{3} \mathrm{OH}$. Only species such as $\mathrm{H}_{2} \mathrm{O}$ and $\mathrm{NH}_{3}$ would have been produced and evaporated, leading to an enhancement of N-bearing organics like $\mathrm{CH}_{3} \mathrm{CN}$. On the other hand, the temperature in the compact ridge region probably fell below $20 \mathrm{~K}$ during the collapse, so there would have been enough solid $\mathrm{CO}$ to be hydrogenated to $\mathrm{CH}_{3} \mathrm{OH}$, giving rise to the O-rich chemistry. Further studies are needed to check whether similar small-scale gradients occur in more distant hot cores.

Lifetime effects and chemical evolution may also explain differences observed between Orion IRc2 and other sources. The Orion $\mathrm{S}$ region just 1.5' from Orion IRc2, for example, is second only to Orion IRc2 in its emission from $\mathrm{SiO}$ and is also a strong $\mathrm{SO}$ emitter. $\mathrm{SO}_{2}$ is extremely weak, however, and the complex molecules that dominate the emission of the Orion hot core are all but absent despite a similar, though slightly decreased, stellar luminosity and outflow intensity. This suggests that Orion S lies at an earlier stage of evolution 
and has not yet had time for large amounts of chemical complexity to evolve (McMullin et al 1993, Groesbeck 1994).

\subsection{IRAS 16293-2422}

The deeply embedded source IRAS 16293-2422 in Ophiuchus $\left(\sim 27 L_{\odot}, d \sim\right.$ $160 \mathrm{pc}$ ) is the best-studied low-mass YSO in terms of chemistry (Mundy et al 1992, Blake et al 1994, van Dishoeck et al 1995). Like many other low-mass objects, it is a binary system, with estimated stellar masses of $\sim 0.5 M_{\odot}$ each at a separation of $5^{\prime \prime}(840 \mathrm{AU})$, located near the center of a large-scale outflow (Walker et al 1990, Mizuno et al 1990). Single-dish line surveys have revealed at least three physically and chemically different components within the $20^{\prime \prime}$ (3000 $\mathrm{AU}$ ) beam: 1. A warm and dense core that is rich in $\mathrm{SiO}, \mathrm{SO}_{2}$, and organics such as $\mathrm{CH}_{3} \mathrm{OH}, \mathrm{HCN}$, and $\mathrm{CH}_{3} \mathrm{CN}$; this region is only 500-1500 AU in size and most likely represents the interaction of the outflow and stellar radiation with the inner part of the circumbinary envelope. 2. A colder circumbinary envelope, $\sim 2000 \mathrm{AU}$ in size, that contains simple, common species such as CS, $\mathrm{HCO}^{+}$, and $\mathrm{H}_{2} \mathrm{CO} .3$. The cold, lower density outer envelope and surrounding cloud core that contains radicals and unsaturated molecules like $\mathrm{CN}, \mathrm{C}_{2} \mathrm{H}$, and $\mathrm{C}_{3} \mathrm{H}_{2}$. Recent interferometric observations of these species at $\sim 6^{\prime \prime}$ resolution by Y Lee and coworkers (Y Lee, LG Mundy, EF van Dishoeck, C Qi, GA Blake, in preparation) confirm this picture, with the strongest emission originating from the compressed gas lying along the outflow lobes. On small scales, the abundances of $\mathrm{HCN}$ and $\mathrm{SO}_{2}$ are enhanced by factors of 5-10 around the southern source $\mathrm{MM} 1$, whereas $\mathrm{SiO}$ and $\mathrm{SO}_{2}$ are increased similarly around the northern MM2 source, which is presumably the more active of the two. The $\mathrm{CH}_{3} \mathrm{OH}$ abundance does not show significant variations on the 500- to 3000-AU scale, which suggests that the release of grain mantles occurs over a large region. As in other sources (Section 7.2), $\mathrm{N}_{2} \mathrm{H}^{+}$appears to avoid the inner envelope. Altogether, this study shows that qualitatively, the same chemical characteristics can be distinguished in both low-mass and high-mass objects. In detail, there certainly are differences, for example in the mechanism(s) for releasing grain mantles. For low-mass objects, the luminosity is too low to radiatively heat the dust except in the innermost $100 \mathrm{AU}$, so release of ice mantles through grain-grain collisions in shocked zones is more likely to dominate.

\section{CHEMISTRY IN CIRCUMSTELLAR DISKS AND RELATION WITH COMETS}

\subsection{Observations}

After the circumstellar envelopes have been dispersed by winds and/or radiation, an optically visible star with an attendant circumstellar disk remains. A large 
fraction of 1- to 5-Myr T Tauri stars are now known to possess disks with masses of $\sim 10^{-3}-10^{-1} M_{\odot}$ and sizes of $\sim 100 \mathrm{AU}$, comparable to those inferred for the primitive solar nebula (Beckwith \& Sargent 1996). Our quantitative understanding of the physical properties of such disks-in particular their radial and vertical temperature and density structures and their gas survival time scaleshas been, until recently, poor. Important steps toward addressing some of these questions have now been taken with millimeter interferometers, which have imaged the gas and dust in over a dozen objects (e.g. Koerner \& Sargent 1995, Dutrey et al 1996, Mundy et al 1996, Hogerheijde et al 1997b; see Sargent 1996 for review). Disks have also recently been detected around intermediatemass Herbig Ae stars (Mannings \& Sargent 1997, Mannings et al 1997). The nature or existence of circumstellar disks around $\mathrm{O}$ and $\mathrm{B}$ stars is uncertain at present.

To date, most of the imaging of disks around T Tauri and Herbig Ae stars has been carried out in various isotopomers of $\mathrm{CO}$ for reasons of sensitivity. A major topic of discussion is the gas-to-dust ratio in such disks, since the $\mathrm{CO}$ data have sometimes been interpreted to yield gas masses that are up to two orders of magnitude lower than those inferred from the continuum emission (Zuckerman et al 1995, Dutrey et al 1996). Several explanations for this discrepancy have been advanced, including an inadequate description of the radiative transfer in the optically thick ${ }^{12} \mathrm{CO}$ line and the possibility that the time scale for gas dissipation is shorter than that of the dust. Another popular explanation is the freeze-out of molecules onto the grains in the cold outer part of the disk at >5-20 AU. However, recent models of flared circumstellar disks heated by radiation from the star indicate a surface layer with temperatures in excess of $100 \mathrm{~K}$ out to $100 \mathrm{AU}$ (Chiang \& Goldreich 1997). Significant quantities of cold gas may also be "hidden" from molecular line observations unless a combination of very small beams and exceptional sensitivity is employed. The amount of gas and dust available within an accretion disk and the time scale over which they are dissipated play major roles in determining what kind of planetary system, if any, can be formed.

Work on the chemical properties of circumstellar disks is only just beginning. An understanding of how various volatile species $\left(\mathrm{H}_{2} \mathrm{O}, \mathrm{CO}, \mathrm{CH}_{4}, \mathrm{NH}_{3}, \mathrm{~N}_{2}\right.$, etc) are distributed in their outer regions is particularly important to examining the connection between interstellar and nebular processes in the formation of icy planetesimals such as comets and Kuiper Belt objects. For three objects, namely DM Tau, GG Tau (Dutrey et al 1997), and TW Hya (Kastner et al 1997), singledish surveys have been carried out in which a number of species $(\mathrm{HCN}, \mathrm{CN}$, $\mathrm{HNC}, \mathrm{H}_{2} \mathrm{CO}, \mathrm{HCO}^{+}$, CS, etc) are detected whose emission intensity is similar to that of ${ }^{13} \mathrm{CO}$ or $\mathrm{C}^{18} \mathrm{O}$, with the higher transitions found to be more intense (Figure 9). These important first data suggest that, at least in appropriate disks, 

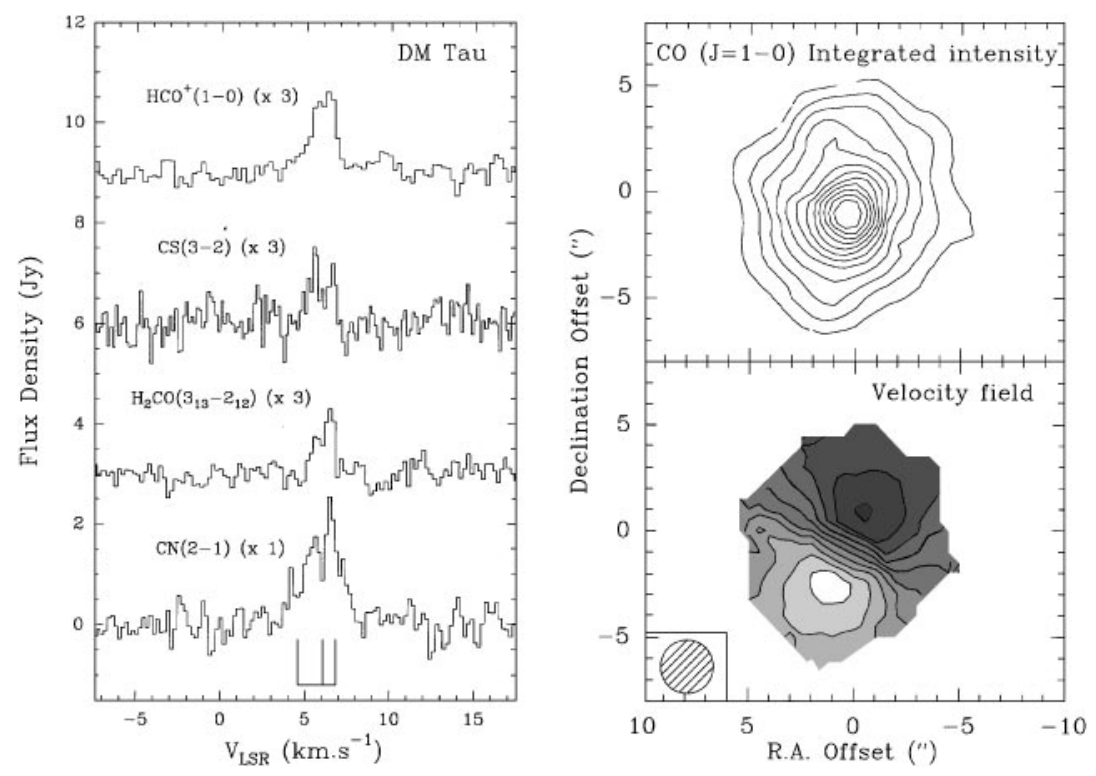

Figure 9 Right: Circumstellar disk around DM Tau as imaged in ${ }^{12} \mathrm{CO} 1-0$ with the IRAM Plateau de Bure interferometer. The velocity field shows blueshifted gas in the southeast, redshifted gas in the northwest, and is consistent with Keplerian rotation. Left: Spectra of various molecules detected in the DM Tau disk with the IRAM 30-m telescope (Dutrey et al 1996, 1997).

chemical studies regarding the nature and variation of the disk composition with radius can now be profitably pursued.

The initial results indicate that both ion-molecule chemistry and photondominated chemistry must contribute to the observed abundances, since the ratios of species such as $\mathrm{CN} / \mathrm{HCN}$ and $\mathrm{HNC} / \mathrm{HCN}$ are too high to be accounted for by quiescent chemical models alone (Dutrey et al 1997, Kastner et al 1997). At abundances appropriate for YSO envelopes, the optical depths of transitions from species such as $\mathrm{HCN}$ and $\mathrm{HCO}^{+}$are sufficiently large that their emission should be comparable to that of ${ }^{12} \mathrm{CO}$ if the gas is well mixed and uniform (MR Hogerheijde, private communication). The fact that they are lower suggests that the sizes of the emitting regions are strongly species dependent owing to chemical transformations or condensation onto grains (Dutrey et al 1997). Blake et al (1992) gave a similar explanation for the absence of strong CS emission from the HL Tau disk, whereas Hogerheijde et al (1997b) presented limits on $\mathrm{HCO}^{+}$in the disk around T Tauri N. A fuller understanding of the details of disk chemistry obviously requires interferometric images of several species in each important chemical family (C-, N-, O-, and S-bearing) with the kind 
of sensitivity, dynamic range, and high spatial resolution $(<100 \mathrm{AU})$ that has only recently become possible.

\subsection{Models}

The observed emission strengths can be compared with those predicted by current models of the chemistry in the outer regions of circumstellar accretion disks (for recent examples, see Aikawa et al 1996, 1997, Bauer et al 1997, Finocchi \& Gail 1997). At large radii, the chemistry is controlled kinetically by two-body gas-phase processes and by adsorption to and desorption from grains. Most of the models assume only thermal desorption and use very cold disk midplane temperatures that are lower than those found in recent self-consistent treatments of the radiative transfer in such objects (Chiang \& Goldreich 1997). A number do not include any direct chemical effects of radiation from the young star, and therefore they maximize the likelihood of depletion onto grain mantles, resulting in too-low abundances compared with observations. The most complete models now include effects such as $\mathrm{X}$ rays, short-lived radio nuclides and cosmic-ray ionization, and photochemistry (Finocchi \& Gail 1997, Glassgold et al 1997, Shu et al 1997), but other important processes, such as radiative transfer (including suprathermal dust blankets, dust versus gas settling, and size sorting) and transport need to be considered in future models.

\subsection{Relation with Comets}

Comets are thought to be among the most primitive bodies left over from the planetesimal building stage of the solar nebula (e.g. Mumma et al 1993, Weissman 1995). The past two years have witnessed two of the most important cometary apparitions of this century, those of C/1996 B2 Hyakutake and C/1995 O1 Hale-Bopp. Thanks to the new facilities at (sub)millimeter and infrared wavelengths, many molecules have been detected for the first time, and the complete list includes $\mathrm{H}_{2} \mathrm{O}, \mathrm{CO}, \mathrm{HCO}^{+}, \mathrm{H}_{2} \mathrm{CO}, \mathrm{CH}_{3} \mathrm{OH}, \mathrm{HCN}, \mathrm{HNC}$, $\mathrm{CH}_{3} \mathrm{CN}, \mathrm{CS}, \mathrm{OCS}, \mathrm{H}_{2} \mathrm{~S}, \mathrm{HC}_{3} \mathrm{~N}, \mathrm{HNCO}, \mathrm{HCOOH}, \mathrm{CH}_{3} \mathrm{CHO}, \mathrm{CO}_{2}, \mathrm{CH}_{4}, \mathrm{C}_{2} \mathrm{H}_{2}$, and $\mathrm{C}_{2} \mathrm{H}_{6}$ (see Bockelée-Morvan 1997, Crovisier 1998 for reviews, and also Biver et al 1997). (A listing of species detected in Hale-Bopp may be found at $<$ http://iraux2.iram.fr/HB/comet.html >.) Most of them are parent molecules originating directly from the cometary ices.

Table 1 compares estimates of the average composition of dust ice mantles in the envelopes around massive young stars with the volatile content of these comets. Within the rather considerable uncertainties of each data set, the abundances agree remarkably well. The abundance ratios of various species (HCN/HNC: Irvine et al 1996; $\mathrm{CO} / \mathrm{CH}_{4}, \mathrm{~N}_{2} / \mathrm{NH}_{3}$ : Womack et al 1992) along with critical isotopic ratios such as D/H (in $\mathrm{HDO} / \mathrm{H}_{2} \mathrm{O}$ or DCN/HCN: BockeléeMorvan et al 1998, Crovisier 1998) are also consistent with those observed in 
star-forming regions. This suggests an evolutionary history in which cometary materials remain at very low temperatures throughout their assemblage and for the bulk of their lives. Such a scenario would be consistent with models in which cometary ices are largely direct interstellar condensates, as has been argued by Greenberg \& Hage (1990) and Greenberg \& Remo (1997). The alternative view that they are dominated by a subsequent generation of processed and recondensed ices (Yamamoto 1985, Lunine et al 1991, Notesco \& Bar-Nun 1996) is less favored at present, although outgassing of the most volatile components such as $\mathrm{CO}$ and $\mathrm{N}_{2}$ is likely to be considerable. Extensively altered planetesimals such as those that would result from a warm nebular or protoJovian formation zone (Lewis \& Prinn 1980) do not have a composition that is compatible with that observed in comets.

\section{CONCLUDING REMARKS}

As illustrated in this review, the chemical composition of matter in dense molecular clouds clearly responds to the enormous changes in physical parameters that accompany star formation. Particular attention has been paid to those species whose abundances are dramatically affected and that may therefore serve as unambiguous chemical and temporal markers. Most of the scenario is based on single-dish data of a few well-studied objects, however. The next step is obviously to obtain more quantitative information at higher spatial resolution on a series of objects of wide ranges of mass and luminosity at different evolutionary stages.

The significant progress made over the past twenty years has been driven by the close interaction between models and increasingly detailed observations. Thanks to equally enormous, and continuing, improvements in computer speed, molecular data storage capacity, and numerical algorithms, modeling efforts are now progressing rapidly beyond the pseudo-time-dependent variety. Full coupling of the chemistry with the (multidimensional) hydrodynamics, including thermal balance equations for the gas and dust as well as a detailed radiative transfer treatment of atomic and molecular transitions, is still a daunting task but may soon be within reach. For both models and analyses of observations, continued laboratory and theoretical work on fundamental molecular data and chemical processes remains essential.

In the future as in the past, a tremendous role will be played by new instrumentation at infrared and (sub)millimeter wavelengths that will open up entirely new avenues of research. The next generation of (sub)millimeter arrays will have vastly larger collecting areas and will operate over wider frequency ranges at much better sites compared with present instruments. They will be able to probe the chemistry on scales of a few AU in the nearest star-forming regions, 
allowing studies ranging from the imaging of chemical gradients within circumstellar accretion disks to the precise delineation of molecule formation within shocks and an interrogation of the molecular complexity in hot cores. High spectral resolution instruments on ground-based 8- to 10-m telescopes and aboard the SOFIA (Stratospheric Observatory for Infrared Astronomy) will provide essential complementary observations, allowing important gas-phase species $\left(\mathrm{H}_{2}\right.$, $\mathrm{CH}_{4}, \mathrm{C}_{2} \mathrm{H}_{2}$ ) and solid-state components to be probed in a large variety of both high- and low-mass objects, complementing space-borne instruments onboard ISO and SIRTF (Space Infrared Telescope Facility). In the far-infrared region, $\mathrm{H}_{2} \mathrm{O}$ is one of the most important species in YSOs whose diagnostic potential is only now starting to be explored. Proper analyses will require heterodyne spectral resolution observations above the atmosphere such as those planned with SWAS, ODIN, and, ultimately, FIRST.

Chemistry is developing into a powerful tool to probe the earliest phases of the formation of low- and high-mass stars. Its richness and diversity also adds considerable insight into studies of the last stages of stellar formation and the earliest stages of planetary growth. The time scales of physical and chemical changes are similar, so their evolution is intimately coupled. Astrochemistry is therefore not only interesting in and of itself, but it also has a great deal to contribute to astrophysics.

\section{ACKNOWLEDGMENTS}

The authors are grateful to all their astrochemical colleagues for sending preprints and reprints of their work and to the dedicated staff at telescopes around the world for making the observations possible. They thank JH Black, P Ehrenfreund, E Herbst, TJ Millar, WA Schutte, AGGM Tielens, and CM Walmsley for many stimulating discussions and comments on the manuscript. Astrochemistry in Leiden is supported by grant 781-76-015 from the Netherlands Foundation for Research in Astronomy and at Caltech by the National Science Foundation, grant AST96-13717, and the National Aeronautics and Space Administration Origins of Solar Systems and Exobiology Programs (grants NAG5-4383 and 3733).

Visit the Annual Reviews home page at http://www.AnnualReviews.org.

Literature Cited

Acord JM, Walmsley CM, Churchwell E. 1997. Ap. J. 475:693-704

Aikawa Y, Miyama SM, Nakano T, Umebayashi

T. 1996. Ap. J. 467:684-97
Aikawa Y, Umebayashi T, Nakano T, Miyama SM. 1997. Ap. J. Lett. 486:L51-L54

Allamandola LJ, Sandford SA, Valero GJ. 1988. Icarus 76:225-52 
Allen M, Robinson GW. 1977. Ap. J. 212:396415

Avery LW, Chiao M. 1996. Ap. J. 463: 642-49

Bachiller R. 1996. Annu. Rev. Astron. Astrophys. 34:111-54

Bachiller R, Perez-Gutierrez M. 1997. Ap. J. Lett. 487:L93-L96

Bakes ELO. 1997. The Astrochemical Evolution of the Interstellar Medium. Vledder: Twin. $106 \mathrm{pp}$.

Baluteau JP, Cox P, Cernicharo J, Pequignot D, Caux E, et al. 1997. Astron. Astrophys. 322:L33-L36

Basu S, Mouschovias TC. 1994. Ap. J. 432:72041

Bates DR, Spitzer L. 1951. Ap. J. 113: 441-63

Bauer I, Finocchi F, Duschl WJ, Gail HP, Schloder JP. 1997. Astron. Astrophys. 317: 273-89

Beckwith SVW, Sargent AI. 1996. Nature 383: 139-44

Beckwith SVW, Staude J, Quetz A, Natta A, eds. 1996. Disks and Outflows Around Young Stars. Berlin: Springer

Bergin EA, Goldsmith PF, Snell RL, Langer WD. 1997a. Ap. J. 482:285-97

Bergin EA, Langer WD. 1997. Ap. J. 486:31628

Bergin EA, Melnick GJ, Neufeld DA. 1998. Ap. $J$. In press

Bergin EA, Ungerechts H, Goldsmith PF, Snell RL, Irvine WM. 1997b. Ap. J. 482:267-84

Bernstein MP, Sandford SA, Allamandola LJ, Chang S, Scharberg MA. 1995. Ap. J. 454: $327-44$

Biver N, Bockelée-Morvan D, Colom P, Crovisier J, Davies JK, et al. 1997. Science 275:1915-18

Blake D, Allamandola L, Sandford S, Hudgins D, Freund F. 1991. Science 254:548-51

Blake GA. 1997. See van Dishoeck 1997, pp. 31-44

Blake GA, Mundy LG, Carlstrom JE, Padin S, Scott SL, et al. 1996. Ap. J. Lett. 472:L49L52

Blake GA, Sandell G, van Dishoeck EF, Groesbeck TD, Mundy LG, Aspin C. 1995. Ap. J. 441:689-701

Blake GA, Sutton EC, Masson CR, Phillips TG. 1986. Ap. J. Suppl. 60:357-74

Blake GA, Sutton EC, Masson CR, Phillips TG. 1987. Ap. J. 315:621-45

Blake GA, van Dishoeck EF, Jansen DJ, Groesbeck T, Mundy LG. 1994. Ap. J. 428:680-92

Blake GA, van Dishoeck EF, Sargent A. 1992. Ap. J. Lett. 391:L99-L103

Bockelée-Morvan D. 1997. See van Dishoeck 1997, pp. 219-35

Bockelée-Morvan D, Gautier D, Lis DC, Young K, Keene J, et al. 1998. Icarus. In press

Boogert ACA, Helmich FP, van Dishoeck EF,
Schutte WA, Tielens AGGM, Whittet DCB. 1998. Astron. Astrophys. In press

Boogert ACA, Schutte WA, Tielens AGGM, Whittet DCB, Helmich FP, et al. 1996. Astron. Astrophys. 315:L377-80

Boss A, Myhill EA. 1995. Ap. J. 451:218-24

Boudin N, Schutte WA, Greenberg JM. 1998. Astron. Astrophys. 331:749-59

Briggs R, Ertem G, Ferris JP, Greenberg JM, McCain PJ, et al. 1992. Mendoza-Gomez CX, Schutte W. 1992. Orig. Life 22:287307

Brown PD, Charnley SB, Millar TJ. 1988. MNRAS 231:409-17

Campbell MF, Butner HM, Harvey PM, Evans NJ, Campbell MB, Sabbey CN. 1995. Ap. J. 454:831-49

Cardelli JA, Meyer DM, Jura M, Savage BD. 1996. Ap. J. 467:334-40

Carr JS, Evans NJ, Lacy JL, Zhou S. 1995. Ap. J. 450:667-90

Casanova S, Montmerle T, Feigelson ED, André P. 1995. Ap. J. 439:752-70

Caselli P, Hartquist TW, Havnes O. 1997. Astron. Astrophys. 322:296-301

Caselli P, Hasegawa TI, Herbst E. 1993. Ap. J. 408:548-58

Caselli P, Hasegawa TI, Herbst E. 1998a. Ap. J. 495:309-16

Caselli P, Walmsley CM, Terzieva R, Herbst E. 1998b. Ap. J. In press

Ceccarelli C, Caux E, White GJ, Molinari S, Furniss I, et al. 1998. Astron. Astrophys. 331:372-82

Ceccarelli C, Hollenbach DJ, Tielens AGGM. 1996. Ap. J. 471:400-26

Cecchi-Pestellini C, Aiello S. 1992. MNRAS 258:125-33

Cernicharo J, González-Alfonso E, Alcolea J, Bachiller R, John D. 1994. Ap. J. Lett. 432:L59-L62

Cernicharo J, Lim T, Cox P, González-Alfonso E, Caux E, et al. 1997. Astron. Astrophys. 323:L25-L28

Cernicharo J, Thum C, Hein H, John D, Garcia P, et al. 1990. Astron. Astrophys. 231:L15L18

Cerqueira AH, de Gouveia Dal Pino EM, Herant M. 1997. Ap. J. Lett. 489:L185-88

Cesaroni R, Churchwell E, Hofner P, Walmsley CM, Kurtz S. 1994. Astron. Astrophys. 288:903-20

Cesaroni R, Felli M, Testi L, Walmsley CM, Olmi L. 1997. Astron. Astrophys. 325:72544

Cesaroni R, Walmsley CM, Churchwell E. 1992. Astron. Astrophys. 276:489-506

Chandler CJ, Carlstrom JE. 1996. Ap. J. 466: 338-51

Chandler CJ, DePree CG. 1995. Ap. J. Lett. 455:L67-L71 
Chandler CJ, Wood DOS. 1997. MNRAS 287: 445-54

Charnley SB. 1997. Ap. J. 481:396-405

Charnley SB. 1998. MNRAS 291:455-60

Charnley SB, Kress ME, Tielens AGGM, Millar TJ. 1995. Ap. J. 448:232-39

Charnley SB, Millar TJ. 1994. MNRAS 270: 570-74

Charnley SB, Tielens AGGM, Millar TJ. 1992. Ap. J. Lett. 399:L71-L74

Chernin LM, Masson CR, Fuller GA. 1994. Ap. J. 436:741-48

Chiang EI, Goldreich P. 1997. Ap. J. 490:36876

Chiar JE, Adamson AJ, Kerr TH, Whittet DCB. 1995. Ap. J. 455:234-43

Chiar JE, Gerakines PA, Whittet DCB, Pendleton YJ, Tielens AGGM, et al. 1998. Ap. J. In press

Choi MH, Evans NJ, Gregersen EM, Wang YS. 1995. Ap. J. 448:742-47

Churchwell E. 1990. Astron. Astrophys. Rev. 2:79-123

Codella C, Testi L, Cesaroni R. 1997. Astron. Astrophys. 325:282-94

Combes F, Wiklind T. 1995. Astron. Astrophys. 303:L61-L64

Combes F, Wiklind T, Nakai N. 1997. Astron. Astrophys. 327:L17-L20

Crovisier J. 1998. In Formation and Evolution of Solids in Space, ed. JM Greenberg. Dordrecht: Kluwer. In press

Cummins SE, Linke RA, Thaddeus P. 1986. Ap. J. Suppl. 60:819-78

Dalgarno A. 1987. In Physical Processes in Interstellar Clouds, ed. G Morfill, MS Scholer, pp. 219-39. Dordrecht: Reidel

Dartois E, d'Hendecourt L, Boulanger F, Jourdain de Muizon M, Breitfellner M, et al. 1998. Astron. Astrophys. 331:651-60

de Boisanger C, Helmich FP, van Dishoeck EF. 1996. Astron. Astrophys. 310:315-27

de Gouveia Dal Pino EM, Birkinshaw M, Benz W. 1996. Ap. J. Lett. 460:L111-14

Dent WRF, Matthews HE, Walther DM. 1995. MNRAS 277:193-209

d'Hendecourt LB, Allamandola LJ, Baas F, Greenberg JM. 1982. Astron. Astrophys. 109: L12-L14

d'Hendecourt LB, Allamandola LJ, Greenberg JM. 1985. Astron. Astrophys. 152:130-50

d'Hendecourt L, Jourdain de Muizon M, Dartois E, Breitfellner M, Ehrenfreund P, et al. 1996. Astron. Astrophys. 315:L365-68

Doty SD, Neufeld DA. 1997. Ap. J. 489: 12242

Draine BT, Lee HM. 1984. Ap. J. 285:89-108

Draine BT, McKee CF. 1993. Annu. Rev. Astron. Astrophys. 31:373-432

Draine BT, Roberge WG, Dalgarno A. 1983. Ap. J. 264:485-507
Duley WW, Williams DA. 1984. Interstellar Chemistry. London: Academic. 251 pp.

Dutrey A, Guilloteau S, Duvert G, Prato L, Simon M, et al. 1996. Astron. Astrophys. 309:493-504

Dutrey A, Guilloteau S, Guélin M. 1997. Astron. Astrophys. 317:L55-L58

Ehrenfreund P, Boogert ACA, Gerakines PA, Tielens AGGM. 1998. J. Chem. Soc. Far. Disc. In press

Ehrenfreund P, Boogert ACA, Gerakines PA, Tielens AGGM, van Dishoeck EF. 1997a. Astron. Astrophys. 328:649-69

Ehrenfreund P, Breukers R, d'Hendecourt L, Greenberg JM. 1992. Astron. Astrophys. 260: 431-36

Ehrenfreund P, d'Hendecourt L, Dartois E, Jourdain de Muizon M, Breitfellner M, et al. 1997b. Icarus 130:1-15

Evans NJ, Lacy JH, Carr JS. 1991. Ap. J. 383:674-92

Finocchi F, Gail H-P. 1997. Astron. Astrophys. 327:825-44

Flower DR, Pineau des Forêts G. 1994. MNRAS 268:724-32

Flower DR, Pineau des Forêts G. 1995. MNRAS 275:1049-56

Flower DR, Pineau des Forêts G, Field D, May PW. 1996. MNRAS 280:447-57

Flower DR, Pineau des Forêts G, Walmsley CM. 1995. Astron. Astrophys. 294:815-24

Frerking MA, Langer WD, Wilson RW. 1982. Ap. J. 262:590-605

Fuller GA, Myers PC, Welch WJ, Goldsmith PF, Langer WD, et al. 1991. Ap. J. 376:135-49

Garay G, Köhnenkamp I, Rodríguez LF. 1996. ESO Messenger 83:31-37

Geballe TR, Oka T. 1996. Nature 384:334-35

Gensheimer PD, Mauersberger R, Wilson TL. 1996. Astron. Astrophys. 314:281-94

Genzel R, Reid MJ, Moran JM, Downes D, Ho PTP. 1982. Ap. J. Lett. 259:L103-7

Genzel R, Stutzki J. 1989. Annu. Rev. Astron. Astrophys. 27:41-85

Gerakines PA, Schutte WA, Ehrenfreund P. 1996. Astron. Astrophys. 312:289-305

Gerin M, Combes F, Wlodarczak G, Jacq T, Guélin M, et al. 1992. Astron. Astrophys. 259:L35-L38

Gerola H, Glassgold AE. 1978. Ap. J. Suppl. $37: 1-25$

Glassgold AE, Najita J, Igea J. 1997. Ap. J. 480:344-50

Gredel R, Lepp S, Dalgarno A, Herbst E. 1989. Ap. J. 347:289-93

Greenberg JM, Hage JI. 1990. Ap. J. 361:26074

Greenberg JM, Li A, Mendoza-Gómez CX, Schutte WA, Gerakines PA. 1995. Ap. J. Lett. 455:L177-80

Greenberg JM, Mendoza-Gómez CX, de Groot 
MS, Breukers R. 1993. See Millar \& Williams 1993, pp. 271-95

Greenberg JM, Remo JL. 1997. Ann. Rev. NY Acad. Sci. 822:96-117

Gregersen EM, Evans NJ, Zhou SD, Choi MH. 1997. Ap. J. 484:256-76

Grim RJA, Greenberg JM, de Groot MS, Baas F, Schutte WA, Schmitt B. 1989. Astron. Astrophys. Suppl. 78:161-86

Groesbeck TD. 1994. PhD thesis. Calif. Inst. Technol., Pasadena, CA

Guilloteau S, Bachiller R, Fuente A, Lucas R. 1992. Astron. Astrophys. 265:L49-L52

Hanawa T, Yamamoto S, Hirahara Y. 1994. Publ. Astron. Soc. Jpn. 420:318-25

Hartigan P, Kenyon SJ, Hartmann L, Strom SE, Edwards S. 1991. Ap. J. 382: 617-35

Hartquist TW, Caselli P, Rawlings JMC, Ruffle DP, Williams DA. 1998. See Hartquist \& Williams 1998. In press

Hartquist TW, Rawlings JMC, Williams DA, Dalgarno A. 1993. Q. J. R. Astron. Soc. 34:213-33

Hartquist TW, Williams DA, eds. 1998. The Molecular Astrophysics of Stars and Galaxies. Oxford: Oxford Univ. Press

Hasegawa TI, Herbst E. 1993a. MNRAS 261: 83-102

Hasegawa TI, Herbst E. 1993b. MNRAS 263: 589-606

Hatchell J, Millar TJ, Rodgers SD. 1998a. Astron. Astrophys. 332:695-702

Hatchell J, Thompson MA, Millar TJ, Macdonald GH. 1998b. Astron. Astrophys. In press

Helmich FP, Millar TJ, van Dishoeck EF. 1998. Astron. Astrophys. Submitted

Helmich FP, van Dishoeck EF. 1997. Astron. Astrophys. Suppl. 124:205-53

Helmich FP, van Dishoeck EF, Black JH, de Graauw T, Beintema DA, et al. 1996a. Astron. Astrophys. 315:L173-76

Helmich FP, van Dishoeck EF, Jansen DJ. 1996b. Astron. Astrophys. 313:657-63

Henning T. 1997. See van Dishoeck 1997, pp. 343-56

Herbst E. 1993. See Millar \& Williams 1993, pp. 183-204

Herbst E. 1995. Annu. Rev. Phys. Chem. 46:2753

Herbst E. 1997. In CO: Twenty-Five Years of Radio Astronomy, ed. WB Latter, SJE Radford, PR Jewell, JG Mangum, J Bally, IAU Symp. 170, pp. 71-78. Dordrecht: Kluwer

Herbst E, Klemperer W. 1973. Ap. J. 185:50533

Herbst E, Leung CM. 1989. Ap. J. Suppl. 69:271-300

Hermsen W, Wilson TL, Walmsley CM, Henkel C. 1988. Astron. Astrophys. 201:285-98

Hirahara Y, Suzuki H, Yamamoto S, Kawaguchi K, Kaifu N, et al. 1992. Ap. J. 394:539-51
Hofner P, Churchwell E. 1996. Astron. Astrophys. Suppl. 120:283-99

Hofner P, Kurtz S, Churchwell E, Walmsley CM, Cesaroni R. 1996. Ap. J. 460:359-71

Hogerheijde MR, van Dishoeck EF, Blake GA, van Langevelde HJ. 1997a. Ap. J. 489:293313

Hogerheijde MR, van Dishoeck EF, Blake GA, van Langevelde HJ. 1998. Ap. J. In press

Hogerheijde MR, van Langevelde HJ, Mundy LG, Blake GA, van Dishoeck EF. 1997b. Ap. J. Lett. 490:L99-L102

Hollenbach DJ. 1997. In Herbig-Haro Objects and the Birth of Low Mass Stars, ed. B Reipurth, IAU Symp. 182, pp. 181-98. Dordrecht: Kluwer

Hollenbach DJ, McKee CF. 1989. Ap. J. 342: 306-36

Hollenbach DJ, Salpeter EE. 1971. Ap. J. 163: 155-64

Hollenbach DJ, Tielens AGGM. 1997. Аnnu. Rev. Astron. Astrophys. 35:179-215

Hollenbach DJ, Tielens AGGM. 1998. Rev. Mod. Phys. In press

Howe DA, Taylor SD, Williams DA. 1996. MNRAS 279:143-51

Hunter TR, Phillips TG, Menten KM. 1997. Ap. J. 478:283-94

Irvine WM, Bockelée-Morvan D, Lis DC, Matthews HE, Biver N, et al. 1996. Nature 383:418-20

Irvine WM, Goldsmith PF, Hjalmarson Å. 1987. In Interstellar Processes, ed. D Hollenbach, HA Thronson, pp. 561-609. Dordrecht: Reidel

Irvine WM, Schloerb FP, Hjalmarson $\AA$, Herbst E. 1985. In Protostars \& Planets II, ed. DC Black, MS Matthews, pp. 579-620. Tucson: Univ. Arizona Press

Jacq T, Walmsley CM, Henkel C, Baudry A, Mauersberger R, et al. 1990. Astron. Astrophys. 228:447-70

Jenniskens P, Baratta GA, Kouchi A, deGroot MS, Greenberg JM, Stazzulla G. 1993. Astron. Astrophys. 273:583-600

Jewell PR, Hollis JM, Lovas FJ, Snyder LE. 1989. Ap. J. Suppl. 70:833-64

Johansson LEB, Andersson C, Elldér J, Friberg P, Hjalmarson $\AA$, et al. 1984. Astron. Astrophys. 130:227-56

Jones AP, Tielens AGGM, Hollenbach DJ, McKee CF. 1994. Ap. J. 433:797-810

Kaiser RI, Roessler K. 1997. Ap. J. 475:144-54

Kaiser RI, Stranges D, Lee YT, Suits AG. 1997. Ap. J. 477:982-89

Karpas Z, Meotner M. 1989. J. Phys. Chem. 93: 1859-63

Kastner JH, Zuckerman B, Weintraub DA, Forveille T. 1997. Science 277:67-71

Kaufman MJ, Hollenbach DJ, Tielens AGGM. 1998. Ap. J. In press 
Kaufman MJ, Neufeld DA. 1996. Ap. J. 456: 611-30

Kelly ML, Macdonald GH, Millar TJ. 1996. MNRAS 279:1210-18

Koerner DW, Sargent AI. 1995. Astron. J. 109: 2138-45

Kress ME, Charnley SB. 1996. In The Role of Dust in Star Formation, ed. HU Käufl, R Siebenmorgen, pp. 313-16. Berlin: Springer Krolik JH, Kallman TR. 1983. Ap. J. 267:61024

Kuan YJ, Snyder LE. 1994. Ap. J. Suppl. 94: 651-76

Kuan YJ, Snyder LE. 1996. Ap. J. 470:9811000

Kuiper TBH, Langer WD, Velusamy T. 1996. Ap. J. 468:761-73

Lacy JH, Baas F, Allamandola LJ, Persson SE, McGregor PJ, et al. 1984. Ap. J. 276:53343

Lacy JH, Evans NJ, Achtermann JM, Bruce DE, Arens JF, Carr JS. 1989. Ap. J. Lett. 342:L43L46

Lacy JH, Carr JS, Evans NJ, Baas F, Achtermann JM, Arens JF. 1991. Ap. J. 376:556-60

Lacy JH, Knacke R, Geballe TR, Tokunaga AT. 1994. Ap. J. Lett. 428:L69-L72

Langer WD. 1985. In Protostars and Planets II, ed. DC Black, MS Matthews, pp. 650-67. Tucson: Univ. Arizona Press

Langer WD, Velusamy T, Xie T. 1996. Ap. J. 468:L41-L44

Le Bourlot J, Pineau des Forêts G, Roueff E. 1995. Astron. Astrophys. 297:251-60

Lee HH, Bettens RPA, Herbst E. 1996a. Astron. Astrophys. Suppl. 119:111-14

Lee HH, Herbst E, Pineau des Forêts G, Roueff E, le Bourlot J. 1996b. Astron. Astrophys. 311:690-707

Leen TM, Graff MM. 1988. Ap. J. 325: 411-16

Léger A, Jura M, Omont A. 1985. Astron. Astrophys. 144:147-60

Lepp S, Dalgarno A. 1996. Astron. Astrophys. 306:L21-L24

Lewis JS, Prinn RG. 1980. Ap. J. 238: 357-64

Li AG, Greenberg JM. 1997. Astron. Astrophys. 323:566-84

Li ZY, Shu FH. 1996. Ap. J. 472:211-24

Liseau R, Ceccarelli C, Larsson B, Nisini B, White GJ, et al. 1996. Astron. Astrophys. 315:L181-84

Liszt HS. 1992. Ap. J. 386:139-42

Lizano S, Cantó J, Garay G, Hollenbach D. 1996. Ap. J. 468:739-48

Loren RB, Wootten A. 1985. Ap. J. 299:947-55

Lunine JI. 1997. Orig. Life 27:205-24

Lunine JI, Engel S, Rizk B, Horanyi M. 1991. Icarus 94:333-44

Macdonald GH, Gibb AG, Habing RJ, Millar TJ. 1996. Astron. Astrophys. Suppl. 119:33367
MacKay DDS. 1995. MNRAS 274:694-700

Maloney PR, Hollenbach DJ, Tielens AGGM. 1996. Ap. J. 466:561-84

Mangum JG, Plambeck RL, Wootten A. 1991. Ap. J. 369:169-74

Mangum JG, Wootten A. 1993. Ap. J. Suppl. 89:123-53

Mangum JG, Wootten A, Barsony M. 1997. In Low Mass Star Formation: From Infall to Outflow, ed. F Malbet, A Castets, IAU Symp. 182 Poster Proc. Grenoble: Obs.

Mannings V, Koerner DW, Sargent AI. 1997. Nature 388:555-57

Mannings V, Sargent AI. 1997. Ap. J. 490:792802

Marcy GW, Butler RP. 1996. Ap. J. 464:L14751

Mardones D, Myers PC, Tafalla M, Willner D, Bachiller R, Garay G. 1997. Ap. J. 489:71933

Marechal P, Pagani L, Langer WD, Castets A. 1997. Astron. Astrophys. 318: 252-55

Martín-Pintado J, Bachiller R, Fuente A. 1992. Astron. Astrophys. 254:315-26

Mathis JS. 1996. Ap. J. 472:643-55

Mayor M, Queloz D. 1995. Nature 378:35559

McGonagle D, Irvine WM. 1997. Ap. J. 477:711-21

McGonagle D, Ziurys LM, Irvine WM, Minh YC. 1990. Ap. J. 359:121-24

McKee CF. 1989. Ap. J. 345:782-801

McMullin JP, Mundy LG, Blake GA. 1993. Ap. J. 405:599-607

McMullin JP, Mundy LG, Blake GA. 1994a. Ap. J. 437:305-16

McMullin JP, Mundy LG, Wilking BA, Hezel T, Blake GA. 1994b. Ap. J. 424: 222-36

Mehringer DM, Snyder LE. 1996. Ap. J. 471:897-902

Mehringer DM, Snyder LE, Miao YT. 1997. Ap. J. Lett. 480:L71-L74

Menten KM. 1997. See van Dishoeck 1997, pp. 163-72

Menten KM, Walmsley CM, Henkel C, Wilson TL. 1988. Astron. Astrophys. 198:253-66

Menten KM, Reid MJ. 1995. Ap. J. Lett. 445:L157-60

Meyer DM. 1997. See van Dishoeck 1997, pp. 407-19

Meyer DM, Cardelli JA, Sofia UJ. 1997. Ap. J. Lett. 490:L103-6

Mezger PG, Sievers AW, Haslam CGT, Kreysa E, Lemke R, et al. 1992. Astron. Astrophys. 256:631-39

Miao YT, Mehringer DM, Kuan YJ, Snyder LE. 1995. Ap. J. Lett. 445:L59-L62

Miao YT, Snyder LE. 1997. Ap. J. Lett. 480: L67-L70

Mikami H, Umemoto T, Yamamoto S, Saito S. 1992. Ap. J. Lett. 392:L87-L90 
Millar TJ. 1990. In Molecular Astrophysics, ed. TW Hartquist, pp. 115-31. Cambridge: Cambridge Univ. Press

Millar TJ. 1997. See van Dishoeck 1997, pp. $75-88$

Millar TJ, Bennett A, Herbst E. 1989. Ap. J. 340:906-20

Millar TJ, Farquhar PRA, Willacy K. 1997a. Astron. Astrophys. Suppl. 121:139-85

Millar TJ, Herbst E, Charnley SB. 1991a. Ap. J. 369:147-56

Millar TJ, Macdonald GH, Gibb AG. 1997b. Astron. Astrophys. 325:1163-73

Millar TJ, Macdonald GH, Habing RJ. 1995. MNRAS 273:25-29

Millar TJ, Rawlings JMC, Bennett A, Brown PD, Charnley SB. 1991b. Astron. Astrophys. Suppl. 87:585-619

Millar TJ, Williams DA, eds. 1993. Dust and Chemistry in Astronomy. Bristol: Inst. Phys. (IOP). 335 pp.

Minh YC, Ohishi M, Roh DG, Ishiguro M, Irvine WM. 1993. Ap. J. 411:773-77

Minh YC, Ziurys LM, Irvine WM, McGonagle D. 1990. Ap. J. 360:136-41

Minh YC, Ziurys LM, Irvine WM, McGonagle D. 1991. Ap. J. 366:192-97

Mitchell GF. 1984a. Ap. J. Suppl. 54:81101

Mitchell GF. 1984b. Ap. J. 287:665-70

Mitchell GF, Maillard JP, Allen M, Beer R, Belcourt K. 1990. Ap. J. 363:554-73

Mizuno A, Fukui Y, Iwata T, Nozawa S, Takano T. 1990. Ap. J. 356:184-94

Moore MH, Donn B, Khanna R, A'Hearn MF. 1983. Icarus 54:388-405

Moriarty-Schieven GH, Wannier PG, Mangum JG, Tamura M, Olmsted VK. 1995. Ap. J. 455:190-201

Motte F, André P, Neri R. 1998. Astron. Astrophys. In press

Mumma MJ, Weissman PR, Stern SA. 1993. In Protostars \& Planets III, ed. EH Levy, J Lunine, pp. 1177-252. Tucson: Univ. Arizona Press

Mundy LG, Looney LW, Erickson W, Grossman A, Welch WJ, et al. 1996. Ap. J. 464:L16972

Mundy LG, McMullin JP. 1997. See van Dishoeck 1997, pp. 183-91

Mundy LG, Wootten HA, Wilking BA, Blake GA, Sargent AI. 1992. Ap. J. 385:306-13

Murata Y, Kawabe R, Ishiguro M, Morita KI, Hasegawa T, Hayashi M. 1992. Publ. Astron. Soc. Jpn. 44:381-89

Myers PC, Benson PJ. 1983. Ap. J. 266:30920

Myers PC, Mardones D, Tafalla M. 1996. Ap. J. Lett. 465:L133-36

Nelson RP, Langer WD. 1997. Ap. J. 482:796826
Neufeld DA, Dalgarno A. 1989a. Ap. J. 340: 869-93

Neufeld DA, Dalgarno A. 1989b. Ap. J. 344: 251-64

Nisini B, Lorenzetti D, Cohen M, Ceccarelli C, Giannini T, et al. 1996. Astron. Astrophys. 315:L321-24

Notesco G, Bar-Nun A. 1996. Icarus 122:11821

Nummelin A. 1996. Millimetre-wave observations of the SgrB2 molecular cloud, Tech. Rep. 256L. Göteborg: Chalmers Univ. Press

Ohashi N, Hayashi M, Kawabe R, Ishiguro M. 1996. Ap. J. 466:317-37

Ohashi N, Kawabe R, Hayashi M, Ishiguro M. 1991. Astron. J. 4102:2054-65

Ohishi M. 1997. See van Dishoeck 1997, pp. 61-74

Ohishi M, Irvine WM, Kaifu N. 1992. In Astrochemistry of Cosmic Phenomena, ed. PD Singh, IAU Symp. 150, pp. 171-77. Dordrecht: Kluwer

Olmi L, Cesaroni R, Neri R, Walmsley CM. 1996. Astron. Astrophys. 315:565-77

Oppenheimer M, Dalgarno A. 1974. Ap. J. 192:29-32

Pagani L, Langer WD, Castets A. 1993. Astron. Astrophys. 274:L13-L16

Palumbo ME, Geballe TR, Tielens AGGM. 1997. Ap. J. 479:839-44

Pauls TA, Wilson TL, Bieging JH, Martin RN. 1983. Astron. Astrophys. 124:23-38

Pelletier G, Pudritz RE. 1992. Ap. J. 394:11738

Pendleton YJ, Sandford SA, Allamandola LJ, Tielens AGGM, Sellgren K. 1994. Ap. J. 437:683-96

Pineau des Forêts G, Roueff E, Schilke P, Flower DR. 1993. MNRAS 262:915-28

Plambeck RL, Wright MCH. 1987. Ap. J. Lett. 317:L101-5

Plume R, Jaffe DT, Evans NJ, Martín-Pintado J, Gomez-Gonzalez J. 1997. Ap. J. 476:73049

Poglitsch A, Herrmann F, Genzel R, Madden SC, Nikola T, et al. 1996. Ap. J. Lett. 462:L43-L47

Prasad SS, Heere KR, Tarafdar SP. 1991. Ap. J. 373:123-36

Prasad SS, Tarafdar SP, Villere KR, Huntress WT. 1987. In Interstellar Processes, ed. D Hollenbach, HA Thronson, pp. 631-66. Dordrecht: Reidel

Pratap P, Dickens JE, Snell RL, Miralles MP, Bergin EA, et al. 1997. Ap. J. 486:86285

Rawlings JMC, Hartquist TW, Menten KM, Williams DA. 1992. MNRAS 255:471-85

Reipurth B, Bertout C, ed. 1997. Herbig-Haro Objects and the Birth of Low Mass Stars, IAU Symp. 182. Dordrecht: Kluwer. 596 pp. 
Rodgers SD, Millar TJ. 1996. MNRAS 280: 1046-54

Ruffle DP, Hartquist TW, Taylor SD, Williams DA. 1997. MNRAS 291:235-40

Sandell G, Knee LBG, Aspin CA, Robson IE, Russell APG. 1994. Astron. Astrophys. 285:L1-L4

Sandford SA, Allamandola LJ. 1993. Ap. J. 417:815-25

Sandford SA, Allamandola LJ, Tielens AGGM, Valero GJ. 1988. Ap. J. 329:498-510

Sargent AI. 1996. In Disks and Outflows from Young Stars, ed. SVW Beckwith, J Staude, A Quetz, A Natta, pp. 1-23. Berlin: Springer

Sargent AI, Welch WJ. 1993. Annu. Rev. Astron. Astrophys. 31:297-43

Schilke P, Groesbeck TD, Blake GA, Phillips TG. 1997a. Ap. J. Suppl. 108:301-37

Schilke P, Walmsley CM, Millar TJ, Henkel C. 1991. Astron. Astrophys. 247:487-96

Schilke P, Walmsley CM, Pineau des Forêts G, Flower DR. 1997b. Astron. Astrophys. 321:293-304

Schilke P, Walmsley CM, Pineau des Forêts G, Roueff E, Flower DR, Guilloteau S. 1992. Astron. Astrophys. 256:595-612

Schmitt B. 1994. In Molecules and Grains in Space, ed. I Nenner, pp. 735-57. New York: Am. Inst. Phys.

Schreyer K, Helmich FP, van Dishoeck EF, Henning T. 1997. Astron. Astrophys. 326:34765

Schutte WA. 1996. In Cosmic Dust Connection, ed. JM Greenberg, pp. 1-42. Dordrecht: Kluwer

Schutte WA. 1998. In The Formation and Evolution of Solids in Space, ed. JM Greenberg. Dordrecht: Kluwer. In press

Schutte WA, Allamandola LJ, Sandford SA. 1993. Icarus 104:118-37

Schutte WA, Greenberg JM. 1991. Astron. Astrophys. 244:190-204

Schutte WA, Greenberg JM. 1997. Astron. Astrophys. 317:L43-L46

Schutte WA, Greenberg JM, van Dishoeck EF, Tielens AGGM, Boogert ACA, Whittet DCB. 1998. Astrophys. Space Sci. In press

Schutte WA, Tielens AGGM, Whittet DCB, Boogert A, Ehrenfreund P, et al. 1996. Astron. Astrophys. 315:L333-36

Shalabiea O, Greenberg JM. 1995a. Astron. Astrophys. 296:779-88

Shalabiea O, Greenberg JM. 1995b. Astron. Astrophys. 303:233-41

Shematovich VI, Shustov BM, Wiebe DS. 1997. MNRAS 292:601-10

Shepherd DS, Churchwell E. 1996. Ap. J. 472: 225-39

Shu FH. 1977. Ap. J. 214:488-97

Shu FH, Adams FC, Lizano S. 1987. Annu. Rev. Astron. Astrophys. 25:23-81
Shu FH, Najita J, Galli D, Ostriker E, Lizano S. 1993. In Protostars \& Planets III, ed. EH Levy, J Lunine, pp. 3-46, Tucson: Univ. Arizona Press

Shu FH, Najita J, Ostriker EC, Shang H. 1995. Ap. J. Lett. 455:L155-58

Shu FH, Shang H, Glassgold AE, Lee T. 1997. Science 277:1475-79

Smith IWM. 1997. See van Dishoeck 1997, pp. 253-69

Smith RG, Sellgren K, Tokunaga AT. 1989. Ap. J. 344:413-26

Snell RL, Loren RB, Plambeck RL. 1980. Ap. J. Lett. 239:L17-L22

Snow TP, Witt AN. 1996. Ap. J. Lett. 468:L65L68

Snyder LE. 1997. Orig. Life 27:115-33

Spaans M, Hogerheijde MR, Mundy LG, van Dishoeck EF. 1995. Ap. J. Lett. 455:L16770

Sternberg A, Yan M, Dalgarno A. 1997. See van Dishoeck 1997, pp. 141-53

Strazzulla G, Baratta GA. 1992. Astron. Astrophys. 266:434-38

Sutton EC, Blake GA, Masson CR, Phillips TG. 1985. Ap. J. Suppl. 58:341-78

Sutton EC, Jaminet PA, Danchi WC, Blake GA. 1991. Ap. J. Suppl. 77:255-85

Sutton EC, Peng R, Danchi WC, Jaminet PA, Sandell G, et al. 1995. Ap. J. Suppl. 97:45596

Suzuki H, Yamamoto S, Ohishi M, Kaifu N, Ishikawa S, et al. 1992. Ap. J. 392:55170

Swade DA. 1989. Ap. J. 345:828-52

Tarafdar SP, Prasad SS, Huntress WT, Villere KR, Black DC. 1985. Ap. J. 289:220-37

Tauber J, Olofsson G, Pilbratt G, Nordh L, Frisk U. 1996. Astron. Astrophys. 308:913-23

Tegler SC, Weintraub DA, Rettig TW, Pendleton YJ, Whittet DCB. 1995. Ap. J. 439:27987

Teixeira TC, Emerson JP, Palumbo ME. 1998. Astron. Astrophys. In press

Tielens AGGM. 1983. Astron. Astrophys. 119: 177-84

Tielens AGGM. 1989. In Interstellar Dust, ed. LJ Allamandola, AGGM Tielens, IAU Symp. 135, pp. 239-62. Dordrecht: Kluwer

Tielens AGGM. 1998. In Formation and Evolution of Solids in Space, ed. JM Greenberg. Dordrecht: Kluwer. In press

Tielens AGGM, Allamandola LJ. 1987. In Interstellar Processes, ed. D Hollenbach, HA Thronson, pp. 379-70. Dordrecht: Kluwer

Tielens AGGM, Charnley SB. 1997. Orig. Life 27:23-51

Tielens AGGM, Hagen W. 1982. Astron. Astrophys. 114:245-60

Tielens AGGM, Tokunaga AT, Geballe TR, Baas F. 1991. Ap. J. 381:181-99 
Tielens AGGM, Whittet DCB. 1997. See van Dishoeck 1997, pp. 45-60

Timmermann R, Poglitsch A, Nikola T, Geis N. 1996. Ap. J. Lett. 460:L65-L68

Turner BE. 1989. Space Sci. Rev. 51:235-37

Turner BE. 1990. Ap. J. Lett. 362:L29-L33

Turner BE. 1991. Ap. J. Suppl. 76:617-86

Turner BE. 1994. Ap. J. 430:727-42

Turner BE. 1996. Ap. J. 468:694-721

Turner BE, Ziurys LM. 1988. In Galactic and Extragalactic Radio Astronomy, ed. GL Verschuur, KI Kellerman, pp. 201-54. Berlin: Springer

Turner JL, Welch WJ. 1984. Ap. J. Lett. 287: L81-L84

Umebayashi T, Nakano T. 1990. MNRAS 243: $103-13$

Ungerechts H, Bergin EA, Goldsmith PF, Irvine WM, Schloerb FP. 1997. Ap. J. 482:245-66

van Dishoeck EF. 1988. In Millimetre and Submillimetre Astronomy, ed. RD Wolstencroft, WB Burton, pp. 117-64. Dordrecht: Reidel van Dishoeck EF, ed. 1997. Molecules in Astrophysics: Probes \& Processes, IAU Symp. 178. Dordrecht: Kluwer. 588 pp.

van Dishoeck EF. 1998a. See Hartquist \& Williams 1998. In press

van Dishoeck EF. 1998b. In Formation and Evolution of Solids in Space, ed. JM Greenberg. Dordrecht: Kluwer. In press

van Dishoeck EF. 1998c. J. Chem. Soc. Far. Disc. In press

van Dishoeck EF, Black JH. 1987. In Physical Processes in Interstellar Clouds, ed. G Morfill, MS Scholer, pp. 241-74. Dordrecht: Reidel

van Dishoeck EF, Black JH. 1988. Ap. J. 334: 771-802

van Dishoeck EF, Blake GA. 1995. Astrophys. Space Sci. 224:237-50

van Dishoeck EF, Blake GA, Draine BT, Lunine JI. 1993. In Protostars \& Planets III, ed. EH Levy, J Lunine, pp. 163-244, Tucson: Univ. Arizona Press

van Dishoeck EF, Blake GA, Jansen DJ, Groesbeck T. 1995. Ap.J. 447:760-82

van Dishoeck EF, Helmich FP. 1996. Astron. Astrophys. 315:L177-80

van Dishoeck EF, Helmich FP, de Graauw T, Black JH, Boogert ACA, et al. 1996. Astron. Astrophys. 315:L349-52

van Dishoeck EF, Helmich FP, Schutte WA, Ehrenfreund P, Lahuis F, et al. 1998. In Star Formation with the ISO Satellite, ed. J Yun, R Liseau, 132:54-66. San Francisco: Astron. Soc. Pac.

van Dishoeck EF, Phillips TG, Keene J, Blake GA. 1992. Astron. Astrophys. 261:L13L16

Walker CK, Carlstrom JE, Bieging JH, Lada CJ, Young ET. 1990. Ap. J. 364:173-77
Walmsley CM. 1992. In Chemistry and Spectroscopy of Interstellar Molecules, ed. DK Bohme, E Herbst, N Kaifu, S Saito, pp. 26772. Tokyo: Univ. Tokyo Press

Walmsley CM, Schilke P. 1993. See Millar \& Williams 1993, pp. 37-52

Ward-Thompson D, Scott PF, Hills RE, André P. 1994. MNRAS 268:276-90

Warin S, Benayoun JJ, Viala YP. 1996a. Astron. Astrophys. 308:535-64

Warin S, Castets A, Langer WD, Wilson RW, Pagani L. 1996b. Astron. Astrophys. 306: 935-46

Watson WD. 1978. Annu. Rev. Astron. Astrophys. 16:585-615

Weissman PR. 1995. Annu. Rev. Astron. Astrophys. 33:327-57

Whittet DCB. 1993. See Millar \& Williams 1993, pp. 9-35

Whittet DCB, Gerakines PA, Tielens AGGM, Adamson AJ, Boogert ACA, et al. 1998. Ap. J. Lett. In press

Whittet DCB, Schutte WA, Tielens AGGM, Boogert ACA, de Graauw T, et al. 1996. Astron. Astrophys. 315:L357-60

Wiesemeyer H, Güsten R, Wink JE, Yorke HW. 1997. Astron. Astrophys. 320:287-99

Willacy K, Rawlings JMC, Williams DA. 1994a. MNRAS 269:921-27

Willacy K, Williams DA, Duley WW. 1994b. MNRAS 267:949-56

Williams DA. 1993. See Millar \& Williams 1993, pp. 143-70

Williams DA, Taylor SD. 1996. Q. J. R. Astron. Soc. 37:565-92

Williams RJR, Dyson JE, Redman MP. 1996. MNRAS 280:667-72

Willner SP, Gillett FC, Herter TL, Jones B, Krassner J, et al. 1982. Ap. J. 253:174-87

Wilson TL, Mehringer DM, Dickel HR. 1995. Astron. Astrophys. 303:840-50

Winnewisser G, Herbst E. 1993. Rep. Prog. Phys. 56:1209-73

Wolkovitch D, Langer WD, Goldsmith PF, Heyer M. 1997. Ap. J. 477:241-64

Womack M, Wyckoff S, Ziurys LM. 1992. Ap. J. 401:728-35

Wood DOS, Churchwell E. 1989. Ap. J. 340: 265-72

Wootten A. 1987. In Astrochemistry, ed. MS Vardya, SP Tarafdar, IAU Symp. 120, pp. 311-19. Dordrecht: Reidel

Wright MCH, Plambeck RL, Mundy LG, Looney LW. 1995. Ap. J. Lett. 455:L18588

Wright MCH, Plambeck RL, Wilner DJ. 1996. Ap. J. 469:216-37

Wyrowski F, Walmsley CM. 1996. Astron. Astrophys. 314:265-74

Xie TL, Allen M, Langer WD. 1995. Ap. J. 440:674-85 
Yamamoto T. 1985. Astron. Astrophys. 142:3136

Yan M, Dalgarno A. 1997. Ap. J. 481:296-301

Yorke HW, Bodenheimer P, Laughlin G. 1995. Ap. J. 443:199-208

Yu KC, Bally J, Devine D. 1997. Ap. J. Lett. 485:L45-L48

Zhou SD, Evans NJ, Kompe C, Walmsley CM. 1993. Ap. J. 404:232-46
Ziurys LM, McGonagle D. 1993. Ap. J. Suppl. 89:155-87

Zmuidzinas J, Blake GA, Carlstrom J, Keene J, Miller D, et al. 1995. Proc. Airborne Astron. Symp., ed. MR Haas, JA Davidson, EF Erickson, pp. 33-40. San Francisco: Astron. Soc. Pac.

Zuckerman B, Forveille T, Kastner JH. 1995. Nature 373:494-96 


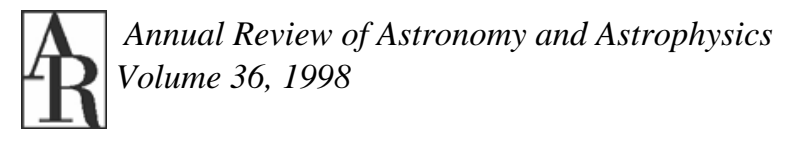

\section{CONTENTS}

Roaming Through Astrophysics, H. C. van de Hulst 1

Type la Supernovae and the Hubble Constant, David Branch 17

Detection of Extrasolar Giant Planets, Geoffrey W. Marcy and R. 57

Paul Butler

First Results from Hipparcos, J. Kovalevsky 99

Radio Emission from Solar Flares, T. S. Bastian, A. O. Benz, and 131

D. E. Gary

Star Formation in Galaxies Along the Hubble Sequence, Robert C. Kennicutt Jr.

189

Herbig Ae/Be Stars, L. B. F. M. Waters, C. Waelkens 233

The Lyman Alpha Forest in the Spectra of Quasistellar Objects, $\quad 267$
Michael Rauch

Chemical Evolution of Star-Forming Regions, Ewine F. van

Dishoeck and Geoffrey A. Blake

Carbon Stars, George Wallerstein and Gillian R. Knapp 369

Dwarf Galaxies of the Local Group, Mario Mateo 435

Astronomical Searches for Earth-Like Planets and Signs of Life, 507

Neville Woolf and J. Roger Angel

Modeling Extragalactic Jets, Attilio Ferrari 539

Simulations of Structure Formation in the Universe, Edmund 599

Bertschinger 